\title{
Vorteilsverbot bzw. Integritäts- und Transparenz- gebot für den Vertrieb von Arzneimitteln der Traditionellen Chinesischen Medizin
}

Rechtliche Rahmenbedingungen zur Bekämpfung der Korruption bei Rabatten und Rückvergütungen im TCM-Bereich

Juana Vasella *

Die Traditionelle Chinesische Medizin (TCM) ist in der Schweiz verbreitet und beliebt. Umso mehr verwundert die Tatsache, dass bloss wenige (spezial-) gesetzliche Regelungen für Alternativmedizin bestehen und sich die Juristen nur vereinzelt mit dieser besonderen Art von asiatischen Arzneimitteln beschäftigen. Soweit ersichtlich, hat sich hierzulande bislang weder die Rechtsprechung noch die Lehre mit der Frage auseinandergesetzt, ob und inwieweit das Gewähren und Annehmen von geldwerten Vorteilen im TCM-Bereich untersagt ist und bestraft werden kann. Ziel des vorliegenden Beitrags ist es, eine Diskussion über die Anwendbarkeit des heilmittelrechtlichen Vorteilsverbotes bzw. des Integritäts- und Transparenzgebots auf den Vertrieb von TCM-Arzneimitteln anzustossen.

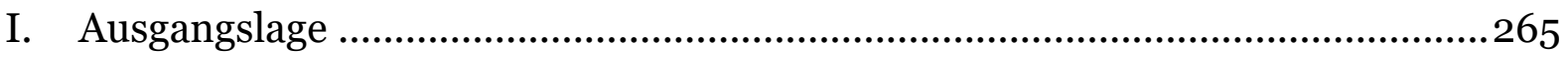

1. TCM-Arzneimittel im Schweizer Heilmittelmarkt ........................................265

2. Kick-backs für Bestellung und Kauf von TCM-Arzneimitteln........................267

3. Offene Rechtsfragen und methodisches Vorgehen.........................................267

II. Korruptionsprävention im TCM-Bereich .........................................................268

1. Relevante Bestimmungen .........................................................................268

2. Aktuelle Revision des Heilmittelrechts ...........................................................269

III. Heilmittelrechtliches Vorteilsverbot de lege lata ................................................269

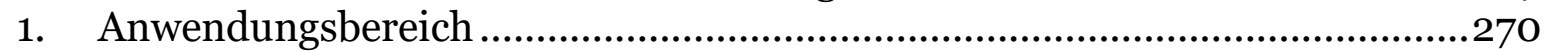

2. Ausnahmetatbestände......................................................................279

IV. Heilmittelrechtliches Integritäts- und Transparenzgebot de lege ferenda .........282

1. Anwendungsbereich .............................................................................28

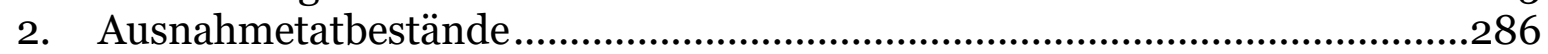

V. Schlussfolgerungen.................................................................................. 288

1. Zusammenfassung der Ergebnisse ........................................................... 288

2. Handlungsempfehlungen für die Praxis ........................................................ 288

Zitiervorschlag: Juana Vasella, Vorteilsverbot bzw. Integritäts- und Transparenzgebot für den Vertrieb von Arzneimitteln der Traditionellen Chinesischen Medizin, in: sui-generis 2017, S. 264

URL: $\quad$ sui-generis.ch/49

DOI: $\quad$ https://doi.org/10.21257/sg.49

* Dr. iur., Rechtsanwältin. Die Autorin ist wissenschaftliche Oberassistentin an der Universität Luzern; daneben ist sie als Konsulentin für MME Legal | Tax | Compliance in Zürich/Zug tätig - in dieser Funktion hat sie Leistungserbringer und Arzneimittelhersteller zur Korruptionsprävention anwaltlich beraten. Sie dankt Dr. iur. Eva-Maria Hiebl, LL.M., für ihre Anregungen und Andrea Voëlin, MLaw, Rechtsanwältin, für die kritische Durchsicht des Manuskripts.

Dieses Werk ist lizenziert unter einer Creative Commons Namensnennung - Weitergabe unter gleichen Bedingungen 4.0 International Lizenz. 


\section{Ausgangslage}

\section{TCM-Arzneimittel im Schweizer Heilmittelmarkt}

1 Traditionelle Chinesische Medizin (TCM) ist ein wahrer Verkaufsschlager - der Schweizer Konsument liebt die fernöstliche Heilkunst. Die aus fünf Therapieformen, den sog. «fünf Säulen der TCM»1, bestehende Heilmethode setzt als ganzheitliches System u.a. auf chinesische Arzneimittel, die hierzulande weit verbreitet sind und in der Bevölkerung eine hohe Akzeptanz geniessen. ${ }^{2}$ Die nationale Gesetzgebung spiegelt diese Entwicklung nicht wider: Der Heilmittelmarkt ist in der Schweiz zwar stark reglementiert, aber spezielle Regelungen in Bezug auf den TCM-Bereich finden sich kaum. Wenn man von dem in der Bundesverfassung (BV) 3 verankerten hohen Stellenwert der alternativen Medizin einmal absehen mag, existieren besondere Vorschriften allenfalls im Hinblick auf die Zulassung von TCM-Präparaten in der Komplementär- und Phytoarzneimittelverordnung (KPAV) 4.5

1 Zu den fünf therapeutischen Verfahren der TCM zählen neben der chinesischen Arzneimitteltherapie («Kräutermedizin») und der Akupunktur inkl. der Erwärmung von Akupunkturpunkten (sog. Moxibustion) auch Massagetechniken (wie Tuina), Bewegungsübungen (wie Qigong) und eine besondere Ernährungslehre (am Wirkprofil der Arzneien ausgerichtete Diätetik); vgl. Schmidt, Traditionelle Chinesische Medizin, Hamburg 2016, Stichwort «TCM»; Porkert, Klinische Chinesische Pharmakologie, Heidelberg 1978, 52.

2 Vgl. Schweizerisches Heilmittelinstitut (Swissmedic), Geschäftsbericht 2016, Bern 2017, 32 (in Bezug auf Komplementärarzneimittel im Allgemeinen).

3 Bundesverfassung der Schweizerischen Eidgenossenschaft vom 18.4.1999 (SR 101).

4 Verordnung über die vereinfachte Zulassung von Komplementär- und Phytoarzneimitteln vom 22.6.2006 (SR 812.212.24).
2 Für rechtliche Fragen zur TCM muss daher regelmässig auf die allgemeinen Vorschriften des Heilmittel- und Gesundheitsrechts zurückgegriffen werden, namentlich auch im Zusammenhang mit der Korruptionsbekämpfung. Relevant sind in diesem Kontext vor allem das Heilmittelgesetz $(\mathrm{HMG})^{6}$, das Krankenversicherungsgesetz (KVG)7, das Medizinalberufegesetz (MedBG) 8 und das Strafgesetzbuch (StGB)9; daneben sind einige heilmittelrechtliche Ausführungsverordnungen wie die Arzneimittelverordnung $(\mathrm{VAM})^{10}$, mehrere standes- bzw. branchenrechtliche Reglemente ${ }^{11}$ und das Obligationenrecht $(\mathrm{OR})^{12} \mathrm{zu}$ beachten.

3 Der Schweizer Heilmittelmarkt lässt sich nach diesen Vorgaben in den Vertrieb von Arzneimitteln und von Medizinprodukten unterteilen. Die Bezeichnung «Heilmittel» dient als Oberbegriff, der sowohl Arzneimittel ${ }^{13}$ als auch Medizin-

5 Vgl. Art. 118a BV sowie Art. 4 Abs. 1 und 3, 25-31 KPAV.

6 Bundesgesetz über Arzneimittel und Medizinprodukte vom 15.12.2000 (SR 812.21).

7 Bundesgesetz über die Krankenversicherung vom 18.3.1994 (SR 832.10).

8 Bundesgesetz über die universitären Medizinalberufe vom 23.6.2006 (SR 811.11).

9 Schweizerisches Strafgesetzbuch vom 21.12.1937 (SR 311.0).

10 Verordnung über die Arzneimittel vom 17.10.2001 (SR 812.212.21).

11 Siehe z.B. Standesordnung der Foederatio Medicorum Helveticorum (StandO FMH) vom 12.12.1996, Standesordnung des Schweizerischen Apothekerverbandes (StandO pharmaSuisse) von November 2009, Verhaltenskodex der pharmazeutischen Industrie in der Schweiz (Pharmakodex) vom 4.12.2003 und Verhaltenskodex der pharmazeutischen Industrie in der Schweiz über die Zusammenarbeit mit Fachkreisen und Patientenorganisationen (PharmaKooperations-Kodex) vom 6.9.2013.

12 Bundesgesetz betreffend die Ergänzung des Schweizerischen Zivilgesetzbuches (Fünfter Teil: Obligationenrecht) vom 30.3.1911 (SR 220).

13 Unter «Arzneimittel» (auch: «Pharmazeutika» oder «Medikamente») versteht man Produkte 
produkte ${ }^{14}$ umfasst (vgl. Art. 2 Abs. 1 lit. a HMG). Die TCM-Präparate stellen eine besondere Form von Arzneimitteln dar. Es handelt sich dabei um Arzneimittel, die aus bestimmten (chinesischen) pflanzlichen, tierischen oder mineralischen Stoffen hergestellt werden.15 TCMArzneimittel können in verschiedenen galenischen Formen verschrieben, abgegeben und angewendet werden. Sie kommen als Einzel- und Formelextrakte sowohl in (halb-)fester als auch in flüssiger Form zum Einsatz - vor allem als Tabletten und Kapseln, Pulver und Granulate sowie Salben und Pasten, aber auch als Sirupe, Tinkturen, Sprays und Rohdrogen. ${ }^{16}$

4 In der Schweiz verfügen mehr als 1'ooo Unternehmen über eine Bewilligung der Schweizerischen Heilmittelbehörde (Swissmedic) für die Herstellung oder Vermittlung von Arzneimitteln oder den Umgang mit kontrollierten Substanzen. ${ }^{17}$ Sie erwirtschaften einen Umsatz von et-

chemischen oder biologischen Ursprungs, die zur medizinischen Einwirkung auf den menschlichen oder tierischen Organismus bestimmt sind oder angepriesen werden, insbesondere zur Erkennung, Verhütung oder Behandlung von Krankheiten, Verletzungen und Behinderungen, wozu auch Blut und Blutprodukte gehören (vgl. Art. 4 Abs. 1 lit. a $\mathrm{HMG}$ ).

14 Als «Medizinprodukte» (auch: «Medical Devices») gelten solche Produkte, die für die medizinische Verwendung bestimmt sind oder angepriesen werden und deren Hauptwirkung nicht durch ein Arzneimittel erreicht wird, einschliesslich Instrumenten, Apparaten, In-vitroDiagnostika, Software und anderen Gegenständen oder Stoffen (vgl. Art. 4 Abs. 1 lit. b HMG).

15 Davon sind rund $85 \%$ pflanzlicher Herkunft, und der Rest ist mineralischer bzw. tierischer Art; vgl. Porkert (Fn. 1), 52.

16 Vgl. Porkert (Fn. 1), 52 f.

17 Ende 2016 lagen insgesamt 1'098 Betriebsbewilligungen vor; vgl. Swissmedic, Geschäftsbericht 2016, 20. Alle Bewilligungsinhaber sind auf der Website der Behörde öffentlich einsehbar. wa 5.6 Mia. CHF pro Jahr ${ }^{18}$ und ca. $4 \%$ des Bruttoinlandprodukts (BIP) ${ }^{19}$, u.a. mit TCM-Präparaten. Von den aktuell fast 8'50o hierzulande zugelassenen Arzneimitteln zählen über 1'700 zur Komplementär- und Phytomedizin ${ }^{20},{ }^{21}$ wozu insbesondere auch die TCM gehört. ${ }^{22}$ Darüber hinaus wird eine Vielzahl an dokumentierten traditionellen chinesischen Stoffen und Zubereitungen ohne Indikation (d.h. Angabe eines Anwendungsgebietes) von Swissmedic anerkannt. ${ }^{23}$ Der TCM-Bereich bildet damit eine wichtige Sparte innerhalb der für den Standort Schweiz volkswirtschaftlich bedeutenden Pharmabranche.
$1 8 \longdiv { \text { Im Jahr } 2 0 1 6 \text { betrug der Umsatz der Schweizer } }$ Pharmaindustrie insgesamt 5'594.8 Mio. CHF (nach Fabrikabgabepreisen); vgl. Verband der forschenden pharmazeutischen Firmen der Schweiz (Interpharma), Pharma-Markt Schweiz, 24. Aufl., Basel 2017, 18.

19 Vgl. Sprecher, Transparenz - Ein Grundprinzip des Rechtsstaats und seine Bedeutung im Gesundheitsrecht, ZSR 135 (2016) II, 139 ff., 214 Fn. 607 (für das Jahr 2013).

20 «Komplementärarzneimittel» umfassen asiatische, homöopathische und anthroposophische Medikamente (vgl. Art. 4 Abs. 1 lit. a KPAV), während «Phytoarzneimittel» als Wirkstoffe ausschliesslich pflanzliche Stoffe oder Zubereitungen enthalten, ohne speziellen Therapierichtungen wie der homöopathischen oder anthroposophischen Medizin zugeordnet zu sein (vgl. Art. 4 Abs. 1 lit. b KPAV); siehe auch Swissmedic, Geschäftsbericht 2016, 33 .

21 Ende 2016 waren insgesamt 8'417 Arzneimittel zugelassen, davon 1'728 Präparate der Alternativmedizin; vgl. Swissmedic, Geschäftsbericht 2016, 20. Listen sämtlicher Human- und Tierarzneimittel mit Zulassung hat Swissmedic auf ihrer Website veröffentlicht.

${ }_{22} \mathrm{Zu}$ dieser dogmatischen Einordnung siehe unten Rz. 28.

23 Diese Stoffe und Zubereitungen werden in der «Traditionellen Asiatischen Stoffliste» («TASListe») aufgeführt; siehe dazu Rz. 30. 


\section{Kick-backs für Bestellung und Kauf von TCM-Arzneimitteln}

5 Wie beim Vertrieb von Heilmitteln im Inund Ausland nicht unüblich, leisten Hersteller, Importeure und Grosshändler auch bei Bestellung und Kauf von TCM häufig Rabatte, Rückvergütungen oder andere Formen von finanziellen bzw. materiellen Abgeltungen («Kick-backs») an Ärzte, Zahn- und Tierärzte, Apotheker, Drogisten, Therapeuten sowie andere Leistungserbringer ${ }^{24}$ im Gesundheitswesen.

6 Bekannt sind etwa "Erfassungsentschädigungen» für die Online-Bestellung von TCM-Arzneimitteln («Variante 1»): Ein Arzt oder Therapeut verschreibt einem Patienten ein TCM-Präparat und ordert es online über eine spezielle Software. Bei Bestellung durch einen Arzt wird das Medikament direkt an den Patienten versandt; bei Bestellung durch einen Therapeuten muss der Patient das Medikament in einer Apotheke seiner Wahl abholen. Die Rechnungsstellung durch den Hersteller oder Grosshändler erfolgt entweder per Sofortrechnung direkt gegenüber dem Patienten oder per periodischer Abrechnung gegenüber dem Arzt bzw. Therapeuten, der seinerseits gegenüber dem Patienten abrechnet. Für die elektronische Bestellung wird dem Leistungserbringer ein bestimmter Prozentsatz des Warenwertes ausgezahlt.

7 Seltener anzutreffen sind «pauschale Vergütungen» oder «Zeilenhonorare» für das Ausstellen von Rezepten für
TCM-Arzneimittel («Variante 2»): So vergütet mancher Apotheker einen bestimmten Prozentsatz des verschriebenen Warenwertes oder eine individuell vereinbarte Summe pro Rezeptzeile (d.h. pro Arzneimittel) an Ärzte, wenn die Patienten ein TCM-Rezept bei ihm einlösen.

\section{Offene Rechtsfragen und methodisches Vorgehen}

8 Sind solche Formen der Kooperation zwischen Pharmaunternehmen und Leistungserbringern bzw. Apothekern und Ärzten bereits eine Spielart von Korruption? Grundsätzlich gilt, dass Kick-backs im Zusammenhang mit dem Vertrieb von Arzneimitteln gesetzlich verboten sind und die Beteiligten behördlich bzw. gerichtlich sanktioniert werden können. Insoweit stellt sich die für die Praxis bedeutsame Frage, ob das Vorteilsverbot (nach altem Recht) bzw. das Integritätsund Transparenzgebot (nach neuem Recht) im Heilmittelbereich nur Sachverhalte mit herkömmlichen Arzneimitteln der Schulmedizin betrifft oder auch Fälle mit den besonderen Präparaten der TCM erfasst.

9 Eine Frage, die trotz der weiten Verbreitung und grossen Beliebtheit von TCM bis anhin nicht geklärt scheint. Die Vermutung liegt nahe, dass solche Fälle, sofern sie überhaupt jemals Gegenstand behördlicher Abklärungen sind, vielfach im Vorfeld eines gerichtlichen Verfahrens in Zusammenarbeit mit den zustän-

24 Dieser Begriff ist dem Krankenversicherungsrecht entlehnt (vgl. Art. 35 Abs. 2 KVG) und wird im Folgenden für solche Personen und Organisationen verwendet, die Arzneimittel verschreiben oder abgeben; siehe dazu Rz. 21. 
digen Verwaltungs- und Strafverfolgungsbehörden bereinigt werden. 25

Die folgenden Ausführungen sollen einen Überblick geben über das heilmittelrechtliche Vorteilsverbot bzw. das heilmittelrechtliche Integritäts- und Transparenzgebot und seine Geltung im TCMBereich: Die einleitenden Betrachtungen beleuchten das komplexe Regelungsgeflecht zur Korruptionsbekämpfung im Gesundheitswesen und die anstehende Reform des Heilmittelrechts (II). Ziel der anschliessenden Analyse ist es, eine Diskussion zu eröffnen über die mögliche Anwendbarkeit der relevanten Bestimmungen auf den Vertrieb von TCMArzneimitteln de lege lata (III) und de lege ferenda (IV). Eine kurze Zusammenfassung (V.1) und praktische Handlungsanweisungen (V.2) runden den Beitrag $a b$.

\section{Korruptionsprävention im TCM-Bereich}

\section{Relevante Bestimmungen}

11 Regelungen betreffend das Gewähren und Annehmen von geldwerten Vorteilen im Zusammenhang mit Arzneimitteln bzw. betreffend die aktive und passive Privatbestechung finden sich in verschiedenen Gesetzen, Verordnungen und Reglementen aus den Gebieten des Heilmittel- und Gesundheitsrechts sowie im Kernstrafrecht und Obligationenrecht. Diese Vorschriften mit unterschiedlichen Anwendungsbereichen und Rechtsfolgen ergeben eine komplexe Re-

25 Ähnlich Gattiker, Arzt und Medizinprodukte, in: Kuhn/Poledna (Hrsg.), Arztrecht in der Praxis, 2. Aufl., Zürich 2007, 495 ff., 503 (zur Abgrenzung zwischen Arzneimitteln und Medizinprodukten). gelungsmaterie. Die wichtigsten Bestimmungen sind:

- Art. 33 HMG («Versprechen und Annehmen geldwerter Vorteile») bzw. Art. 55 und 56 revHMG («Integrität» und «Transparenzpflicht»);

- Art. 56 Abs. 3 und 4 KVG bzw. Art. 56 Abs. 3, $3^{\text {bis }}$ und 4 revKVG («Wirtschaftlichkeit der Leistungen»);

- Art. 40 MedBG («Berufspflichten»);

- Art. $322^{\text {octies }}$ und $322^{\text {novies }}$ StGB («Bestechen» und «Sich bestechen lassen»);

- Art. 400 OR («Rechenschaftsablegung und Herausgabe»);

- Art. 36-39 StandO FMH («Entschädigung für die Zuweisung von Patienten oder Patientinnen», «Entschädigung für wissenschaftliche Studien», "Annahme von Geschenken» und «Fortbildungsveranstaltungen und Sponsoring»), Ziff. 4.6 lit. c und d StandO pharmaSuisse («Werbung und Verhalten auf dem Markt») sowie der Pharmakodex und der Pharma-Kooperations-Kodex.

12 Die vorliegende Untersuchung fokussiert auf die Strafbarkeit nach den Dreh- und Angel-Normen, dem sog. «heilmittelrechtlichen Vorteilsverbot» gemäss Art. 33 HMG bzw. dem sog. «heilmittelrechtlichen Integritäts- und Transparenzgebot» gemäss Art. 55 f. revHMG. ${ }^{26}$ Das regulatorische Ziel dieser Bestimmung ist es, die Beeinflussung von Fachpersonen, die Arzneimittel verschreiben

$26 \overline{\text { Ausführlich zu den anderen Vorgaben Vasella, }}$ Das heilmittelrechtliche Vorteilsverbot, Zürich 2016, 392 ff., 404 ff., 485 ff. und 536 ff. (m.w.H.). 
oder abgeben, bei ihrer therapeutischen Entscheidung für oder gegen bestimmte Medikamente durch finanzielle bzw. materielle Anreize zu unterbinden. Die Behandlung von Patienten soll objektiv und unabhängig erfolgen, d.h. ausschliesslich nach medizinisch-pharmazeutischen Gesichtspunkten sowie nach dem Stand von Wissenschaft und Technik.27 Die Verbotsnorm verfolgt einen gesundheitspolizeilichen Zweck; geschütztes Rechtsgut ist die öffentliche Gesundheit. ${ }^{28}$
Botschaft Heilmittelgesetz (HMG) vom 1.3.1999, BBl 1999 III, 3453 ff., 3518; BGE 140 II 520, 531 E. 5.2.1; Urteil des Bundesgerichts 2P.32/2006 und 2A.56/2006 vom 16.11.2006, E. II.3.3; Urteil 2P.169/2006 und 2A.401/2006 vom 20.9.2007, E. II.3.3; Urteil 2C_92/2011 vom 12.4.2012, E. 3.9.1; Urteil des Bundesverwaltungsgerichts C-669/2008 vom 17.12.2010, E. 4.1.2; Schweizerisches Heilmittelinstitut (Swissmedic), Zulässigkeit von Rabatten, Swissmedic Journal 2003, 980 ff., 980 f., 983, 985; dass., Zum Verbot des Versprechens und Annehmens geldwerter Vorteile, Swissmedic Journal 2006, 20 ff., 27; Eggenberger Stöckli, Arzt und Heilmittel, in: Kuhn/Poledna (Hrsg.), Arztrecht in der Praxis, 2. Aufl., Zürich 2007, 457 ff., 486; Fellmann, Arzt als Unternehmer, in: Poledna/Jacobs (Hrsg.), Gesundheitsrecht im wettbewerblichen Umfeld, Zürich 2010, 135 ff., 138 Rz. 8; Saxer, in: Jaisli/Eichenberger/Richli (Hrsg.), Heilmittelgesetz (HMG), Basler Kommentar, Basel 2006, Art. 33 N 6, 26; Vasella (Fn. 26), 7, 63, 66.

28 Vgl. Urteil des Bundesgerichts 2P.32/2006 und 2A.56/2006 vom 16.11.2006, E. II.3.3; Urteil des Bundesverwaltungsgerichts C-669/2008 vom 17.12.2010, E. 6.3.1; Eggenberger Stöckli, Geldwerte Vorteile von bescheidenem Wert, PharmR 2008, 457 ff., 458; Saxer, BSK HMG (Fn. 27), Art. 33 N 6; ders., Korruption im Arzneimittelhandel, AJP 2002, 1463 ff., 1466; ders., Das Vorteilsverbot gemäss Art. 33 HMG, in: Eichenberger/Poledna (Hrsg.), Das neue Heilmittelgesetz, Zürich 2004, 113 ff., 118; Richli, Regelungsschwerpunkte des Heilmittelgesetzes, in: Eichenberger/Poledna (Hrsg.), Das neue Heilmittelgesetz, Zürich 2004, 47 ff., 51; Vasella (Fn. 26), 66 ff., $71,72$.

\section{Aktuelle Revision des Heilmittelrechts}

13 Die Gesetzgebung zu geldwerten Vorteilen im Zusammenhang mit dem Verschreiben, Abgeben und Anwenden von Arzneimitteln ist im Umbruch. Eine grundlegende Reform wurde bereits im Frühjahr 2016 vom Parlament im Rahmen der ordentlichen Revision des Heilmittelgesetzes («HMG-Revision, 2. Etappe») verabschiedet.29 Die Gesetzesänderungen sollen durch den Bundesrat mithilfe von angepassten und neuen Ausführungsbestimmungen auf Verordnungsebene präzisiert werden. Die Vernehmlassung zur umfassenden Revision des Verordnungsrechts («Heilmittelverordnungspaket, HMV IV») läuft noch bis Herbst 2017.30 Die neuen Regelungen sollen voraussichtlich Anfang 2019 zusammen mit dem revidierten Heilmittelgesetz in Kraft treten. ${ }^{1}$

\section{Heilmittelrechtliches Vorteilsverbot de lege lata}

14 Art. 33 HMG verbietet das Versprechen und Annehmen geldwerter Vorteile.

15 Ein Verstoss gegen Art. 33 HMG kann mit verwaltungsrechtlichen Massnahmen sanktioniert werden (Art. $66 \mathrm{HMG}$ ). Die strafrechtlichen Sanktionen, die der Ge-

29 Die Schlussabstimmung über das revidierte Heilmittelgesetz (und Krankenversicherungsgesetz) erfolgte am 18.3.2016. Die Reform beinhaltet auch eine Änderung von Art. $56 \mathrm{KVG}$, der um einen Abs. $3^{\text {bis }}$ ergänzt wird. Siehe dazu BBl 2016, $1953 \mathrm{ff}$.

3o Das Vernehmlassungsverfahren wird vom 21.6.2017 bis 20.10.2017 durchgeführt.

31 Zum Inhalt und Zeitplan der Revision siehe die online verfügbaren Informationen auf der Website des Bundesamtes für Gesundheit (BAG): Das Inkrafttreten ist für 1.1.2019 geplant; der definitive Termin kann erst nach Auswertung der Vernehmlassungsergebnisse bestimmt werden. 
setzgeber ebenfalls vorgesehen hat (Art. $87 \mathrm{HMG}$ ), wurden während der letzten Jahre in der Praxis nicht mehr ausgesprochen, da das Bundesgericht diese Strafbestimmung im Hinblick auf das heilmittelrechtliche Vorteilsverbot als zu unbestimmt bewertet hat. $3^{2}$

16 Regelungsinhalt und -umfang von Art. 33 HMG, insbesondere von Abs. 3, sind seit dem Inkrafttreten im Jahre 2002 umstritten, was eine gewisse Rechtsunsicherheit zur Folge hat. Die hierzu ergangenen höchstrichterlichen Urteile33 brachten zwar Klarheit in gewissen Punkten, andere Aspekte blieben jedoch offen. Das gab Anlass zur erwähnten Gesetzesreform.

\section{Anwendungsbereich}

\section{a) Allgemeine Auslegung}

Der sachliche Anwendungsbereich beschränkt sich auf die Heilmittelart der Arzneimittel im Sinne von Art. 4 Abs. 1 lit. a HMG.34 Im Bereich der Arzneimittel gilt Art. 33 HMG grundsätzlich umfassend, d.h. die Verbotsnorm greift nach ihrer jetzigen Fassung unabhängig davon, ob die betreffenden Medikamente verschreibungspflichtig oder kassenpflichtig sind:

Die Verschreibungspflicht bildet aus teleologischen Gründen kein taugliches Abgrenzungskriterium. Die Anwendbar-

32 Urteil des Bundesgerichts 6B_771/2011 vom 11.12.2012, E. 2.

33 BGE 140 II 520; Urteile des Bundesgerichts 2C_92/2011 vom 12.4.2012; 6B_771/2011 vom 11.12.2012; 2C_853/2014 und 2C_934/2014 vom 29.9.2015.

34 Medizinprodukte gemäss Art. 4 Abs. 1 lit. b HMG werden nicht erfasst; vgl. Vasella (Fn. 26), 75, 79 f. (m.w.H.). keit der Verbotsnorm zur Auflösung von (möglichen) Interessenkonflikten kann nicht von der Einteilung der Arzneimittel in die fünf Abgabekategorien A-E abgeleitet werden. Denn diese Einordnung, die sich an der Gefährlichkeit der einzelnen Präparate orientiert, wirkt sich nur auf die daran anknüpfende Rezeptpflicht aus.35 Eine begründete Ausnahme besteht hinsichtlich der frei verkäuflichen Medikamente der Abgabekategorie E, da diese nicht zwingend von Fachpersonen abgegeben werden müssen. 36

19 Die Kassenpflicht kann ebenfalls nicht ausschlaggebend sein für die Abgrenzung des Anwendungsbereichs. Die Pflicht zur Übernahme der Kosten für ein Medikament durch die obligatorische Krankenpflegeversicherung wird durch die «Spezialitätenliste» $(« \mathrm{SL} ») 37 \quad$ begründet. 38

35 Die verschreibungspflichtigen Medikamente gemäss Art. 23 Abs. 1, 24 HMG werden in die Abgabekategorien A und B unterteilt (Art. 23 und 24 VAM); die rezeptfreien Medikamente gemäss Art. 23 Abs. 1 und 2, 25 HMG gliedern sich in die Abgabekategorien C und D (Art. 25 und 26 VAM) sowie E (Art. 27 VAM).

36 Präparate der Kategorie E unterliegen keiner Beratungspflicht, weshalb sie auch von medizinischen bzw. pharmazeutischen Laien an Endverbraucher abgegeben werden dürfen. Nach Sinn und Zweck von Art. 33 HMG kann das heilmittelrechtliche Vorteilsverbot deshalb allein auf Pharmazeutika der Kategorien A-D anwendbar sein; so Vasella (Fn. 26), 81 ff., 85. Diese Auslegung ist umstritten: a.A. Urteil des Bundesverwaltungsgerichts C-669/2008 vom 17.12.2010, E. 4.6.1 f.; Urteil C-1663/2007 vom 28.6.2011, E. 4.1.1; gl.M. Swissmedic, Swissmedic Journal 2006 (Fn. 27), 28 f.; Eggenberger Stöckli, Rabatte, PharmR 2007, 393 ff., 393 Fn. 11; dies., PharmR 2008, 458 Fn. 13.

37 Die aktuelle Spezialitätenliste kann auf der Homepage des Bundesamtes für Gesundheit (www.spezialitaetenliste.ch) eingesehen werden

38 Weiterführend Eugster, Krankenversicherung, in: Meyer (Hrsg.), Soziale Sicherheit, Schweizerisches Bundesverwaltungsrecht, Bd. XIV, 3. Aufl., Basel 2016, 337 ff., N 204; Kieser, Heilmittel, in: Poledna/Kieser (Hrsg.), Gesundheitsrecht, Schweizerisches Bundesverwaltungsrecht, Bd. VIII, Basel 
Diese Liste enthält die zugelassenen pharmazeutischen Spezialitäten und konfektionierten Arzneimittel in Form von Originalpräparaten und Generika, jeweils inklusive ihrer amtlich festgesetzten Höchstpreise. 39 Die Regelung der auf SL-Arzneimittel beschränkten Kostenübernahme durch die Versicherer vermag nichts darüber auszusagen, ob für das Verschreiben und Abgeben von Medikamenten, die nicht auf der SL-Liste stehen, geldwerte Vorteile gewährt oder angenommen werden dürfen.40 Denn es kann nicht der Wille des Gesetzgebers sein, dass fast zwei Drittel aller in der Schweiz zugelassenen Arzneimittel nicht erfasst sind. 41

Der persönliche Anwendungsbereich unterscheidet zwischen einer aktiven und einer passiven Seite, d.h. Vorteilsgebern und Vorteilsnehmern:

Auf der Nehmerseite bedarf es einer Beteiligung von verschreibungs- und/ oder abgabeberechtigten Personen oder Organisationen. Bei den Vorteilsnehmern handelt sich somit um Leistungserbringer im Sinne von Art. 35 Abs. 2 KVG, die beruflich bzw. gewerbsmässig

2005, 135 ff., N 4; Marcuard, Preisbildung bei Arzneimitteln, Soziale Sicherheit 2001, 69 ff., 69 f.; Maurer, Das neue Krankenversicherungsrecht, Basel 1996, 91; Poledna/Berger, Öffentliches Gesundheitsrecht, Bern 2002, N 458.

39 Die Grundversicherung vergütet auch die Kosten für komplementärmedizinische Arzneimittel, die auf der SL-Liste aufgeführt sind (vgl. Art. 52 Abs. 1 lit. b KVG).

40 Vgl. Saxer, AJP 2002 (Fn. 28), 1468; Vasella (Fn. 26), $84 \mathrm{f}$.

41 Ende 2016 waren 3'237 von 8'417 zugelassenen Arzneimitteln in der Spezialitätenliste aufgeführt (nach der Zulassungsnummer von Swissmedic). Siehe auch Interpharma, Pharma-Markt Schweiz 2016, 80 f. und 84 f., wo von 2'948 SLMedikamenten die Rede ist (nach der Gammendefinition des BAG).
Medikamente verordnen oder überlassen. ${ }^{22}$ Gemeint sind sowohl universitäre Medizinalpersonen (z.B. Ärzte, Zahnund Tierärzte, Apotheker und Chiropraktoren [vgl. Art. 2 Abs. 1 MedBG]) als auch weitere medizinische und pharmazeutische Fachpersonen (z.B. Drogisten, Hebammen, Dentalhygieniker, Rettungssanitäter und nicht-ärztliche Komplementärmediziner [vgl. Art. 2 Abs. 2 MedBG]) sowie die sie beschäftigenden Organisationen.43 Dabei spielt es im Rahmen von Art. 33 HMG keine Rolle, ob sie im öffentlichen oder privaten Gesundheitswesen tätig sind.

22 Für die Geberseite sieht das Gesetz keine bestimmten Voraussetzungen vor. Als Vorteilsgeber kommen demnach alle natürlichen und juristischen Personen in Betracht, die den verschreibungs- und abgabeberechtigten Personen oder Organisationen geldwerte Vorteile gewähren, anbieten oder versprechen. Dabei bezieht sich Art. $33 \mathrm{HMG}$ aber nur auf diejenigen Dritten in der Vertriebskette, die direkt gegenüber Leistungserbringern - und nicht nur gegenüber Konsumenten - auftreten. 44 Diese Voraussetzung erfüllt jeder, der im Bereich des Arzneimittelhandels im weitesten Sinne tätig ist, d.h. Medikamente vertreibt bzw. deren Vertrieb fördert (vgl. Art. 4 Abs. 1 lit. e

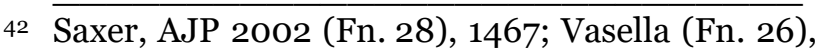
$87 \mathrm{f}$.

43 Vgl. Urteil des Bundesverwaltungsgerichts C669/2008 vom 17.12.2010, E. 4.3.1; Schmidt, Pharmakommunikation - Information oder Werbung?, in: Dörr/Michel (Hrsg.), Biomedizinrecht, Zürich/St. Gallen 2007, 371 ff., 375; Vasella (Fn. 26), $90 \mathrm{f}$.

44 Saxer, AJP 2002 (Fn. 28), 1467; Tagmann, Anreize selbstdispensierender Ärzte, Vergünstigungen auszuhandeln, Managed Care 2004, 22 ff., 22; Vasella (Fn. 26), 86. 
$\mathrm{HMG}$ ). Es bedarf darüber hinaus keiner weitergehenden Sondereigenschaft. 45

Verboten sind geldwerte Vorteile im Arzneimittelvertrieb zu Gunsten von verschreibungs- und abgabeberechtigten Personen und Organisationen. Dieser Begriff wird weit ausgelegt:

24 Als geldwerter Vorteil im Sinne von Art. 33 HMG gilt jede Zuwendung, die ohne überzeugenden Rechtsgrund oder angemessene Gegenleistung erfolgt sowie eine direkte und objektiv messbare finanzielle, wirtschaftliche oder andere materielle Besserstellung bewirkt durch Leistungen und Gegenstände, für die normalerweise ein Preis bezahlt wird. 46 Hierbei kann es sich um Geldleistungen oder Sachwerte, den Erlass von Schulden oder den Verzicht auf die Geltendmachung von Forderungen handeln. 47 Rabatte, Rückvergütungen und andere Preisreduktionen oder Abgeltungen stellen grundsätzlich geldwerte Vorteile im Sinne der Norm dar. 48 Ideelle bzw. immaterielle Vorteile, wie z.B. eine berufliche oder gesellschaftliche Besserstellung des Zuwendungsempfängers, werden hingegen nicht erfasst. 49

45 Urteil des Bundesverwaltungsgerichts C669/2008 vom 17.12.2010, E. 4.2; Vasella (Fn. 26), 86.

46 Saxer, BSK HMG (Fn. 27), Art. 33 N 20 f.; ders., Vorteilsverbot (Fn. 28), $130 \mathrm{f}$.

47 BGE 140 II 520, 532 E. 5.2.2; Eggenberger Stöckli, Arzt (Fn. 27), 486.

48 Vgl. Urteil des Bundesverwaltungsgerichts C669/2008 vom 17.12.2010, E. 4.4; Eggenberger Stöckli, Arzt (Fn. 27), 486.

49 Eggenberger Stöckli, Arzt (Fn. 27), 486; dies., PharmR 2008, 458; dies., Werbung für Heilmittel, in: Poledna (Hrsg.), Gesundheit und Werbung, Zürich 2005, 61 ff., 66; FAEH, Arzneimittelwerbung im europäischen und schweizerischen Recht, Freiburg i.U. 2009, 32 mit Fn. 186; Saxer, BSK HMG (Fn. 27), Art. 33 N 20 ff.; ders., AJP 2002 (Fn. 28), 1468; Vasella (Fn. 26), 99 f.

\section{b) Anwendbarkeit im TCM-Bereich}

25 Die Ärzte, Therapeuten und Apotheker, die in den Genuss der Erfassungsentschädigungen, pauschalen Vergütungen und Zeilenhonorare kommen, sind Medizinalpersonen mit der Berechtigung, Arzneimittel (abgestuft je nach Abgabekategorie) an Patienten bzw. Kunden abzugeben und - im Falle der Ärzte - ihnen auch zu verschreiben. Damit fallen sie in den persönlichen Anwendungsbereich von Art. 33 HMG.

26 Fraglich ist, ob der sachliche Anwendungsbereich von Art. 33 HMG auch im Zusammenhang mit der Verschreibung und Abgabe von Arzneimitteln aus dem TCM-Bereich berührt ist. Behörden und Gerichte haben sich bislang, soweit ersichtlich, noch nicht mit der Frage auseinandergesetzt, wie TCM-Präparate im Lichte des Art. 33 HMG zu bewerten sind. Die konkrete rechtliche Einordnung ist in der Praxis noch offen, wie Anfragen beim Schweizerischen Heilmittelinstitut (Swissmedic) und beim Bundesamt für Gesundheit (BAG) ergeben haben.50 Es bleibt daher zu erörtern, welche Argumente für bzw. gegen eine Anwendbarkeit des heilmittelrechtlichen Vorteilsverbotes auf Arzneimittel der TCM sprechen.

Es kommen verschiedene Abgrenzungskriterien in Betracht: (1) die Zulassung bzw. Zulassungspflicht und (2) die Verwendungsfertigkeit eines Arzneimittels. Die Verschreibungspflicht und die Kassenpflicht von Medikamenten stellen demgegenüber keine geeigneten Unter-

50 Die schriftlichen Rückmeldungen datieren auf den 6.3.2017 und 14.3.2017 (Swissmedic) sowie auf den 16.3.2017 (BAG). 
scheidungsmerkmale dar, wie bereits aufgezeigt wurde. 51

Vorab ist festzuhalten, dass im Hinblick auf die Zulassungspflicht und die Verwendungsfertigkeit zwischen unterschiedlichen Formen von TCMPräparaten, die zu den asiatischen Arzneimitteln und damit zur Komplementärmedizin gehören (vgl. Art. 4 Abs. 1 lit. a und 3 lit. a und b KPAV), zu differenzieren ist:

Zur TCM zählen solche Arzneimittel, die in einer öffentlichen Apotheke oder Spitalapotheke für eine bestimmte Person oder einen bestimmten Personenkreis hergestellt werden, was ad hoc (individuelle Bestellung) oder defekturmässig (auf Vorrat) auf der Grundlage der Materia Medica China und unter Verwendung bestimmter Pflanzen bzw. Pflanzenteile, Pilze, Mineralien oder Tierprodukte erfolgt (formula magistralis).52 Solche TCM-Magistralrezepturen aus einzelnen Komponenten bzw. Stoffen werden unterschieden in Rezepturen, d.h. Eigenherstellung auf individueller Basis in Ausführung einer ärztlichen Verschreibung oder auf Verlangen durch einen Patienten, und Defekturen, d.h. Eigenherstellung in Form von Mehrfachanfertigungen mit Lagerung kleinerer Chargen und festgelegten Höchstmengen.53 Die Magistralrezepturen der TCM sind zwar verwendungsfertig, benötigen aber keine

51 Siehe Rz. $18 \mathrm{f}$.

52 Vgl. Urteil des Bundesgerichts 6B_526/2011 vom 20.3.2012, E. 1.1, 1.3.1 f.

53 Vgl. Urteil des Bundesgerichts 6B_526/2011 vom 20.3.2012, E. 1.1, 1.3.1 f. Zur weiteren Unterscheidung nach standardisierten und individuellen Magistralrezepturen siehe z.B. Gloor/Thoma/Fluhr, Dermatologische Externatherapie, Berlin/Heidelberg 2000, 5 ff.; Höger, Kinderdermatologie, 3. Aufl., Stuttgart 2011, 34.
Zulassung von Swissmedic (vgl. Art. 9 Abs. 2 lit. a HMG).54

30 Von den mehr als 12'ooo Mitteln der Materia Medica China sind nur etwa 600 in der «Chinesischen Pharmakopöe 2005" («PPRC 05») mit Monographien beschrieben und rund 500 regelmässig im Einsatz.55 Darüber hinaus listet Anhang Nr. III zur PPRC 05 weitere 155 Mittel auf, welche in Spezialitäten, d.h. in auf dem Markt verfügbaren Kombinationsund Einzelmitteln, verwendet werden. Nur die in der PPRC o5 aufgeführten Mittel bilden die Grundlage für die «Traditionelle Asiatische Stoffliste» («TASListe») gemäss Anhang 6 zur KPAV. Diese Liste wird von Swissmedic veröffentlicht und stuft die dokumentierten traditionellen asiatischen Stoffe grundsätzlich in die Abgabekategorie $\mathrm{C}$ ein. Einige wenige Stoffe sind aus Gründen der Arzneimittelsicherheit in die Abgabekategorie B eingeteilt; 56 andere Stoffe fallen in die Abgabekategorie D.57 Die auf der TAS-Liste registrierten Mittel sind als ganze Gebinde einzelner Komponenten

$54 \overline{\text { Vgl. Urteil des Bundesgerichts 6B_526/2011 vom }}$ 20.3.2012, E. 1.1, 1.3.1. Die TCMMagistralrezepturen unterstehen aber der Aufsicht des Kantonsapothekers (vgl. Art. 58 Abs. 1, 83 Abs. 1 HMG und Art. 31 Abs. 1 und 2 lit. b VAM); siehe auch BGE 140 II 520, 530 E. 5.1 (m.w.N.).

55 Vgl. Bühlmann, Rohdrogen, Granulate oder flüssige Extrakte, Schweiz Z Ganzheitsmed 2016, 80 ff., 80 .

56 In diesen Fällen wird meist gleichzeitig eine Ausnahme für externe oder orale Anwendungen vorgesehen und damit eine Einordnung in die Abgabekategorie $\mathrm{C}$ vorgenommen; vgl. Jost, Alternativen zu Art. 27 HMG für Therapeuten, Kurzgutachten vom 1.12.2016, Bern 2016, 9.

57 Das ist der Fall, wenn sie in bereits zugelassenen asiatischen Arzneimitteln enthalten sind und davon auszugehen ist, dass die abgebenden Drogisten über die erforderlichen Kenntnisse für eine sichere Empfehlung verfügen; vgl. Jost, Kurzgutachten (Fn. 56), 9. 
verkehrsfähig und handelbar. Die TCMSpezialitäten der Chinesischen Pharmakopöe (d.h. nach dem Chinesischen Arzneibuch), welche Wirkstoffe aus dieser Liste enthalten, müssen als verwendungsfertige Komplementärarzneimittel von Swissmedic zugelassen werden; allerdings genügt ein vereinfachtes Zulassungsverfahren bzw. die Zulassung im Meldeverfahren (vgl. Art. 14 Abs. 1 lit. b und 15 HMG i.V.m. Art. 1 und 4 Abs. 1 lit. a KPAV).

31 Stellt man für den sachlichen Geltungsbereich von Art. 33 HMG auf die Zulassung bzw. Zulassungspflicht von Medikamenten $\mathrm{ab}$, wären im TCM-Bereich nur die Spezialitäten, nicht aber die Magistralrezepturen vom heilmittelrechtlichen Vorteilsverbot erfasst.

32 Für eine solche Auslegung liesse sich vortragen, dass die Zulassung grundsätzlich eine Voraussetzung für das Inverkehrbringen eines Arzneimittels ist (vgl. Art. 9 Abs. 1 Satz 1 HMG) und die Verbotsnorm das lautere Verhalten von Fachpersonen im Zusammenhang mit dem Vertrieb von auf dem Markt erhältlichen Medikamenten sicherstellen soll. Gegen eine derart einschränkende Lesart sprechen indes einige gewichtige Argumente.

33 So beschränkt sich der gesamte 5. Abschnitt über «Werbung und Preisvergleiche» (Art. 31-33 HMG), der auch das Vorteilsverbot enthält, in der Formulierung nicht auf «zugelassene» Präparate, sondern verwendet umfassend den Begriff «Arzneimittel». Auch in der Arzneimittel-Werbeverordnung (AWV)58,

58 Verordnung über die Arzneimittelwerbung vom 17.10.2001 (SR 812.212.5). die sich insbesondere auf Art. 31-33 HMG stützt (vgl. Ingress der AWV) und ganz allgemein auf «Arzneimittel» bezieht, ist keine Begrenzung des Anwendungsbereichs nach der Zulassungsqualität von Medikamenten vorgesehen. Insoweit ist auch auf die (weit gefasste) Legaldefinition in Art. 4 Abs. 1 lit. a HMG $\mathrm{zu}$ verweisen. Sowohl Gesetz als auch Ausführungsverordnung differenzieren somit in ihrem Wortlaut nicht nach den jeweiligen Voraussetzungen für das Inverkehrbringen von Arzneimitteln.

34 Ferner lässt sich die Systematik des Heilmittelgesetzes gegen eine beschränkende Auslegung anbringen. Denn die anderen beiden Werbebestimmungen, Art. 31 und $32 \mathrm{HMG}$, gelten ausdrücklich auch für Arzneimittel ohne Zulassung (vgl. z.B. Art. 31 Abs. 1 lit. a HMG: «alle Arten von Arzneimitteln»; Art. 32 Abs. 1 lit. c HMG: «Arzneimittel, die in der Schweiz nicht in Verkehr gebracht werden dürfen»). Es ist nicht ersichtlich, warum der ebenfalls in den 5. Abschnitt eingegliederte Art. 33 HMG enger ausgelegt werden sollte.

35 Ausserdem stellt sich die Frage nach der durch Art. 33 HMG bezweckten Auflösung von (möglichen) Interessenkonflikten für medizinisch-pharmazeutische Fachpersonen bei allen Arzneimitteln, seien sie zulassungspflichtig (vgl. Art. 9 Abs. $1 \mathrm{HMG}$ ) oder zulassungsbefreit (vgl. Art. 9 Abs. 2 HMG) bzw. nicht zugelassen (vgl. z.B. Art. 20 Abs. 2 lit. b HMG). Das Problem möglicher Interessenkollisionen tritt bei fehlender Zulassung letztlich noch deutlicher zu Tage, da zulassungsbefreite bzw. nicht zugelassene Arzneimittel - anders als zugelassene Arzneimittel - nicht über eine Patienteninformation verfügen 
(z.B. als Faltprospekt, Booklet oder Kombination von Etikette und Packungsbeilage), die nach der ArzneimittelZulassungsverordnung (AMZV)59 für einen Marktzugang vorausgesetzt wird (vgl. Art. 14 AMZV i.V.m. Anhang 5). Denn in jenen Fällen ist die Asymmetrie des Wissensstandes und der Erfahrungswerte zwischen Fachperson und Patient mangels Zulassung und damit einhergehender Informationen zuhanden der Konsumenten noch stärker ausgeprägt, so dass die Behandlung de lege artis umso mehr von den (allein sachlichen Kriterien verpflichteten und seitens aussenstehender Dritter unbeeinflussten) Therapieentscheidungen der Medizinalperson abhängt. ${ }^{60}$ Eine Ausnahme für zulassungsbefreite bzw. nicht zugelassene Medikamente ist daher auch aus teleologischen Erwägungen zu verneinen.

36 Schliesslich lässt sich eine Einschränkung des sachlichen Anwendungsbereichs nach der Zulassung bzw. Zulassungspflicht von Arzneimitteln ebenso wenig mit einer historischen Auslegung anhand der Gesetzesmaterialien begründen. ${ }^{61}$

37 Zieht man für die Abgrenzung des sachlichen Geltungsbereichs von Art. 33 HMG stattdessen die Verwendungsfertigkeit von Medikamenten heran, unterliegen im TCM-Bereich sowohl die Spezialitäten

$59 \overline{\text { Verordnung über die Anforderungen an die } \mathrm{Zu}-}$ lassung von Arzneimitteln vom 9.11.2001 (SR 812.212.22).

6o $\mathrm{Zu}$ diesem Informationsgefälle siehe Botschaft Änderung Heilmittelgesetz (HMG) vom 7.11.2012, BBl 2013, 1 ff., 30, 78; Schüpbach Eastus, Artikel 33 HMG, in: Hettich/Kohler (Hrsg.), St. Galler Tagung zum Pharmarecht, St. Gallen 2010, 117 ff., 147 f.; Sprecher, ZSR 135 (2016) II (Fn. 19), 199 f.; Vasella (Fn. 26), 88 f., 566 (m.w.H.).

61 Vgl. Botschaft HMG, BBl 1999 III, 3518 f. als auch die Magistralrezepturen dem heilmittelrechtlichen Vorteilsverbot.

38 Diese Auslegung gebietet der Schutzzweck der spezialgesetzlichen Verbotsnorm, welche die öffentliche Gesundheit bewahren soll, die auch durch verwendungsfertige Arzneimittel der Alternativmedizin gefährdet sein kann. Denn selbst wenn ein solches Medikament (noch) nicht zugelassen wurde bzw. nicht zulassungspflichtig ist, aber Patienten aufgrund der Verwendungsfertigkeit (bereits) damit therapiert werden bzw. (dereinst) behandelt werden können, besteht die abstrakte Gefahr, dass eine entsprechende Anwendung deren physische und psychische Gesundheit beeinträchtigt.

39 Überdies sprechen die Verfassungsgrundlage (vgl. Art. 118 Abs. 1 und 2 lit. a BV: Gesundheitsschutz) und die Zielrichtung (vgl. Art. 1 Abs. 2 lit. b HMG: Arzneimittelsicherheit und Patientenschutz) des gesamten Heilmittelgesetzes dagegen, dass von Art. 33 HMG fachfremde Einflüsse durch geldwerte Vorteile sehr wohl im Zusammenhang mit geprüften und zugelassenen Arzneimitteln erfasst sein sollen (etwa bei rezeptpflichtigen Medikamenten der Abgabekategorien A und B), nicht aber solche im Zusammenhang mit Arzneimitteln, die zwar verwendungsfertig sind, jedoch nicht vorgängig in einem Zulassungsverfahren von Swissmedic auf Qualität, Sicherheit und Wirksamkeit geprüft wurden (etwa bei defekturmässig hergestellten Magistralrezepturen oder nicht zugelassenen Medikamenten im Spitalbereich).

40 Dieses weite Verständnis legt auch das Vollzugsrecht zu den Art. 31-33 HMG in der Arzneimittel-Werbeverordnung na- 
he, welche explizit die (Fach-)Werbung für «verwendungsfertige» Arzneimittel regelt (vgl. Art. 1 Abs. 1 AWV). Der Begriff der «Verwendungsfertigkeit» beschreibt dabei eine bestimmte Stufe der Arzneimittelproduktion, nämlich den Abschluss der Herstellung im Sinne von Art. 4 Abs. 1 lit. c HMG. Gemeint sind ausschliesslich Endprodukte des Herstellungsprozesses und nicht blosse Rohstof$\mathrm{fe}$, Ausgangsmaterialien, Zwischenprodukte o.ä. ${ }^{62}$ Diese Bestimmung sagt hingegen nichts aus über die unterschiedlichen Voraussetzungen des nachfolgenden Inverkehrbringens der verwendungsfertigen Arzneimittel, wie sie in Art. 9 HMG festgelegt sind (d.h. Grundsatz der Zulassungspflicht nach Abs. 1 und Zulassungsbefreiung als Ausnahme nach Abs. 2). ${ }^{63}$

41 Mit Blick auf die Ausführungsverordnung ist sodann zu bedenken, dass es in Art. 33 HMG ganz allgemein um Fachwerbung für Arzneimittel im Sinne von Art. 2 lit. a und c AWV geht, d.h. um jegliche Werbemassnahmen gegenüber Medizinalpersonen in Form von geldwerten Vorteilen für das Verschreiben bzw. Abgeben von Medikamenten.64 Dement-

$62 \overline{\text { Vgl. Botschaft HMG, BBl } 1999 \text { III, 3488, 3490 }}$ und 3495; Eggenberger Stöckli, ArzneimittelWerbeverordnung (AWV), Stämpflis Handkommentar, Bern 2006, Art. 1 N 19.

63 Ausführlich dazu Mosimann/Schott, in: Jaisli/Eichenberger/Richli (Hrsg.), Heilmittelgesetz (HMG), Basler Kommentar, Basel 2006, Art. 9 $\mathrm{N} 32 \mathrm{ff}$.

64 Zur Fachwerbung (in Abgrenzung zur Publikumswerbung) siehe Eggenberger Stöckli, Arzt (Fn. 27), 482 ff.; dies., SHK AWV, Art. 2 N 40 ff.; dies., Werbung, 65 f., 78 ff.; Eichenberger/Marti/Straub, Die Regulierung der Arzneimittelwerbung, recht 2003, 225 ff., 232 ff., 235 ff.; Faeh, Arzneimittelwerbung, 21 f., 24 ff., 28 ff., 34 ff.; Poledna/do Canto, Werbeverbote für Heilmittel und das Auskündigungsrecht der Ärzte, Jusletter vom 30.1.2012, Rz. 14, 22 ff.; sprechend erfolgt der Gesetzesvollzug durch die Behörden bis anhin im Hinblick auf alle verwendungsfertigen Arzneimittel, wird doch Art. 33 HMG von Swissmedic auch auf verwendungsfertige, aber zulassungsbefreite oder nicht zugelassene Medikamente angewandt. 65 Das ist etwa der Fall bei der Lieferung von (bisher) nicht zugelassenen Arzneimittelmustern (vgl. Art. 4 lit. i und 10 AWV) oder dem Sponsoring von zulassungsbefreiten Arzneimitteln für klinische Versuche (vgl. Art. 9 Abs. 2 lit. d HMG).

42 Ergebnis: Wenngleich es keine gefestigte Praxis zur Anwendbarkeit von Art. 33 HMG im TCM-Bereich gibt, ist davon auszugehen, dass das Gewähren und Annehmen von geldwerten Vorteilen im $\mathrm{Zu}$ sammenhang mit dem Vertrieb von TCM-Arzneimitteln behördlich und gerichtlich verfolgt und sanktioniert würde. Solch einem restriktiven Vorgehen wäre zuzustimmen. Denn nach der hier vertretenen Ansicht unterfallen verwendungsfertige TCM-Präparate unabhängig davon, ob sie zugelassen bzw. zulassungspflichtig sind, dem sachlichen Anwendungsbereich von Art. 33 HMG.

43 Der Staat ist mit Blick auf das geschützte Rechtsgut der öffentlichen Gesundheit und auf die Effizienz entsprechender Schutzmassnahmen gehalten, die Patienten auch beim Verschreiben, Abgeben und Anwenden von Arzneimitteln der Al-

Schmidt, Pharmakommunikation (Fn. 43), 375 ff., 379 ff., 383 f., 389; dies., Die Abgrenzung zwischen Werbung und Information im Arzneimittelrecht, recht 2007, 244 ff., 244 und 247; Vasella (Fn. 26), 19, $88 \mathrm{f}$.

65 Vgl. Schweizerisches Heilmittelinstitut (Swissmedic), Musterpackungen in der Fachwerbung, Swissmedic Journal 2010, 29 ff., 30. 
ternativmedizin davor zu bewahren, dass sich die behandelnden Fachpersonen nicht allein von medizinischpharmazeutischen Kriterien leiten lassen, sondern in ihre Therapieentscheidungen finanziell-materielle Aspekte (unbewusst) einfliessen lassen. ${ }^{66}$ Ebenso wenig vermag das Gebot der Verhältnismässigkeit staatlicher Eingriffe tragfähige Argumente gegen einen Einbezug von TCM-Präparaten zu liefern, denn das heilmittelrechtliche Vorteilsverbot beeinträchtigt die freiheitliche Wirtschaftsordnung und den freien Wettbewerb nicht. ${ }^{67}$

44 Daraus folgt, dass das heilmittelrechtliche Vorteilsverbot in der aktuellen Fassung auch auf TCM-Arzneimittel anzuwenden ist, und zwar nicht nur auf die (erleichtert) zugelassenen Spezialitäten, sondern auch auf die (individuell) hergestellten Magistralrezepturen. Dies ist insofern von Bedeutung, als die TCMMagistralrezepturen weitaus mehr als die 600 in der PPRC 05 umschriebenen Komponenten erfassen können, sondern vielmehr eine Kombination der über 12 '0oo in der Materia Medica China bekannten Mittel erlauben. Für die Herstellung und für die Abgabe der Magistralrezepturen ist ein «doppeltes» ärztliches Rezept erforderlich, was den Ärzten beim Vertrieb dieser TCM-Arzneimittel eine Schlüsselrolle zuweist - die besonders anfällig sein kann für Korruption.

66 Zum Schutzzweck der Norm siehe bereits Rz. 12 und die dort in Fn. 27 angegebenen Fundstellen.

67 Zur Rechtfertigung des staatlichen Eingriffs in die Wirtschaftsfreiheit (vgl. Art. $27 \mathrm{BV}$ ) durch Art. 33 HMG siehe Vasella (Fn. 26), 16 ff., 32 f.
Bereich. Eine Ausnahme liegt - wie bei den herkömmlichen (Fertig-)Arzneimitteln der Schulmedizin - nur bei solchen TCMPräparaten vor, die der Abgabekategorie E angehören.

46 Im Hinblick auf die geldwerten Vorteile zu Gunsten der Leistungserbringer ist nach den oben vorgestellten Fallkonstellationen zu unterscheiden:

47 Die geldwerten Vorteile im Fall der Online-Bestellungen bestehen in den Erfassungsentschädigungen, welche die Leistungserbringer prozentual je nach Warenwert der verschriebenen TCMArzneimittel erhalten. Solche Vergütungen stellen finanzielle Zuwendungen dar, welche auch im TCM-Bereich grundsätzlich verboten sind. Denn sie sind in dem Sinne vom Umsatz abhängig, als sie proportional zur Anzahl der online bestellten Arzneimittel anwachsen. Die sich aus dem Geschäftsmodell ergebende enge wirtschaftliche Beziehung zwischen dem Abgabeverhalten der Ärzte bzw. Therapeuten und Apotheker und der hierfür von ihnen bezogenen Vergütung ist geeignet, einen Anreiz für therapiefremde Mengenausweitungen zu setzen. ${ }^{68}$ Solche umsatzbezogenen Rückvergütungen sollen durch Art. 33 HMG gerade unterbunden werden.

48 Für die Zulässigkeit ist daher ausschlaggebend, ob hier ein bestimmter Rechtsgrund oder eine angemessene Gegenleistung vorliegt. Es ist kein gesetzlich geregelter Rechtsgrund ersichtlich, aber eine Gegenleistung könnte darin zu sehen sein, dass die Ärzte bzw. Therapeuten die TCM-Arzneimittel beim Hersteller online über eine spezielle Software bestellen.

68 Vgl. BGE 140 II 520, 532 E. 5.3.3. 
Mithilfe dieser elektronischen Rezepte verringern sie den personellen und logistischen Aufwand für den Produzenten im Vergleich zu schriftlichen Bestellungen mittels Email oder Fax. Die Erfassungsentschädigungen an die Leistungserbringer können deren Aufwand abgelten, der bei Online-Bestellungen aufgrund der Erfassung und Übermittlung von Daten sowie der Kosten für die spezielle Software anfällt, sodass eine Gegenleistung der Ärzte bzw. Therapeuten bejaht werden kann.

Im Einzelfall ist die rechtliche Zulässigkeit folglich davon abhängig, ob die jeweiligen Rückvergütungen in Höhe eines bestimmten Warenwertes noch das Erfordernis der Angemessenheit erfüllen. Eine klare Obergrenze für die Abgrenzung zwischen erlaubten und verbotenen Zuwendungen kann mangels einer gefestigten Rechtsprechung nicht genannt werden. Erfassungsentschädigungen für Online-Bestellungen dürfen aber auch im TCM-Bereich nur sehr geringfügig ausfallen.

$\mathrm{Zu}$ beachten ist ferner, dass das Bundesgericht und Swissmedic bereits entschieden haben, dass die Ausstellung eines Rezepts eine medizinische Leistung darstellt, welche nach dem «Tarif Médical» $(« T A R M E D » 69)$ als Teil der über den Zeittarif entschädigten Grundkonsultation über die obligatorische Krankenversicherung abgegolten wird, d.h. der Arzt darf nicht nochmals finanziell entschä-

69 Die TARMED-Grundvergütung erfolgt nach der aufgewendeten Zeit und gilt für alle ärztlichen Tätigkeiten, die nicht als spezifische Tarifpositionen separat im TARMED erfasst sind; vgl. BGE 140 II 520, 532 E. 5·3.2. digt werden..$^{\circ}$ Etwas anderes gilt nur, wenn anhand einer Vollkostenkalkulation dargelegt werden kann, dass und in welchem Ausmass der konkrete Aufwand im jeweiligen Einzelfall die Abgeltung nach TARMED übersteigt.71

51 Diese rechtliche Beurteilung für Ärzte lässt sich auf Apotheker und Therapeuten übertragen, denn die Leistungen jener Medizinalpersonen werden von den Krankenversicherungen ebenfalls über feste Tarife abgegolten («Tarifuertrag $L O A I V » 72$ und «Tarif 590»73). Auch ihre Einzelleistungstarife beruhen auf einer gesamtschweizerisch vereinbarten einheitlichen Tarifstruktur (vgl. Art. 43 Abs. 5 KVG).

52 Fazit: Erfassungsentschädigungen für Online-Bestellungen im TCM-Bereich dürfen eine minimale Höhe nicht überschreiten. Darüber hinaus muss der (nur sehr geringe) Aufwand, der zusätzlich zu den nach TARMED abgegoltenen Leistungen entschädigt wird, ganz konkret durch Vollkostenkalkulation nachgewiesen werden. Es wäre darzulegen, inwiefern die elektronische Ausstellung eines Rezepts gegenüber der schriftlichen Verschreibung einen Mehraufwand bedeutet und dass dieser Mehraufwand nicht bereits durch den TARMED abgedeckt ist. Ein solcher Nachweis dürfte in der Praxis schwierig zu erbringen bzw. mit erhebli-

$7 0 \longdiv { \text { Vgl. BGE } 1 4 0 \text { II 520, } 5 3 2 \text { E. 5.3.1 ff.; ebenso Kie- } }$ ser/Poledna, Grenzen finanzieller Interessen von Medizinalpersonen, AJP 2008, 420 ff., $429 \mathrm{f}$.

71 Vgl. BGE 140 II 520, 532 E. 5·3.1.

72 Der «Tarifstruktur-Vertrag LOA IV/1 betreffend Tarifstruktur für Apothekerleistungen (LOA IV/1)» datiert auf den 1.1.2016.

73 Der «Tarif Nr. 590 für ambulante komplementärmedizinische Leistungen nach VVG» ist ab 1.1.2018 verbindlich; bis dahin gilt eine Übergangsfrist, in der anderweitig aufgestellte Rechnungen noch toleriert werden. 
chem organisatorischen Zusatzaufwand verbunden sein. In allen anderen Fällen läge ein geldwerter Vorteil im Sinne von Art. $33 \mathrm{HMG}$ vor. die geldwerten Vorteile in den pauschalen Vergütungen und Zeilenhonoraren, welche die Ärzte für das Ausstellen der später eingelösten Rezepte erhalten. Dabei ist eine mögliche Beeinflussung der ärztlichen Therapieentscheidungen und damit eine Gefahr für die Patientensicherheit zu gewärtigen, weil die pauschal berechneten Rückvergütungen gewisse Anreize zur Verordnung von TCMArzneimitteln setzen.74 Dieses Anreizsystem steht im Widerspruch zum Schutzgedanken von Art. 33 HMG und ist damit grundsätzlich verboten.

54 Bei diesem Geschäftsmodell ist ebenfalls keine gesetzliche Bestimmung ersichtlich, worauf sich die finanziellen Zuwendungen als rechtmässig zurückführen liessen. Ebenso wenig erbringen die Ärzte irgendwelche (wenn auch nur minimale) Arbeiten, die durch die Rückvergütungen entlohnt würden, weshalb auch kein vertragliches Austauschverhältnis vorliegt.

Die Rechtsprechung hat sich bereits mit einem Zeilenhonorar in Höhe von $5 \mathrm{CHF}$ pro Zeile/Arzneimittel auseinandergesetzt und entschieden, dass eine solche Zuwendung nicht angemessen ausgestaltet ist.75 Denn bei guter Organisation benötigt ein Arzt kaum mehr als eine Minute für das Ausfüllen eines Rezepts, sodass

74 Kieser/Poledna, AJP 2008 (Fn. 70), 429 f.; Vasella (Fn. 26), 56.

75 Vgl. Urteil des Bundesgerichts 2C_477/2012 vom 7.7.2014, SV D, H und J (z.T. nicht abgedruckt in BGE 140 II 520 ff.) und E. 5.3.1 ff. und 5.3.5. er in dieser Zeit bspw. für drei Zeilen pro Rezeptur insgesamt 15 CHF verdienen kann. ${ }^{76}$

56 Abgesehen von diesen kritischen Punkten handelt es sich beim Verschreiben von Arzneimitteln um medizinische Leistungen, die den Ärzten bereits über die TARMED-Grundvergütung nach aufgewendeter Zeit abgegolten werden. Ein davon nicht erfasster Mehraufwand müsste konkret ausgewiesen werden.

57 Fazit: Bei den pauschalen Vergütungen in Höhe eines bestimmten Prozentsatzes des Warenwertes oder einer vereinbarten Summe pro Rezeptzeile handelt es sich um geldwerte Vorteile, die gemäss Art. 33 HMG auch im TCM-Bereich grundsätzlich untersagt sind. Ein überzeugender Rechtsgrund oder eine angemessene Gegenleistung sind in diesen Fällen nicht erkennbar.

\section{Ausnahmetatbestände}

\section{a) Allgemeine Auslegung}

58 Art. 33 Abs. 3 HMG sieht bestimmte Tatbestandskonstellationen vor, in denen das Gewähren und Annehmen von geldwerten Vorteilen im Zusammenhang mit dem Verschreiben und Abgeben von Arzneimitteln ausnahmsweise erlaubt ist. Auslegung und Anwendung der beiden Ausnahmetatbestände sind umstritten.

59 Nach Art. 33 Abs. 3 lit. a HMG besteht eine «partielle Bagatellausnahme»77 für geringwertige Vorteile:

76 Vgl. Kieser/Poledna, AJP 2008 (Fn. 70), 429; Vasella (Fn. 26), 56.

77 Vasella (Fn. 26), 104, 107 f.; siehe auch Saxer, AJP 2002 (Fn. 28), 1471. 
60 Die genaue Höhe der erlaubten Zuwendungen, die ein Vorteil von bescheidenem Wert sein müssen, hat der Gesetzgeber nicht explizit festgelegt. Bisher fehlt es auch, soweit ersichtlich, an einschlägiger Judikatur. In der Literatur 78 und in Behörden-Publikationen 79 wird die Obergrenze von 300 CHF pro Vorteilsgeber und Vorteilsnehmer bzw. Geschäftsbeziehung sowie Jahr angegeben. Allerdings erscheint es angebracht, bei der Auslegung nicht von einer starren Grenze auszugehen. Vielmehr ist die Frage nach der Erfüllung dieses Tatbestandsmerkmals anhand der konkreten Umstände des Einzelfalls zu beantworten. ${ }^{80}$ Dabei kann der Betrag von 300 CHF jedoch als Richtwert dienen. ${ }^{81}$ Exemplarisch genannt seien kleine Werbegeschenke, die kostenlose einfache Verpflegung an einer Fachveranstaltung und sozial übliche Präsente als Dank für eine gute Zusammenarbeit oder anlässlich eines Betriebsjubiläums. ${ }^{82}$

61 Nicht jede geringwertige Zuwendung unterfällt dem Erlaubnistatbestand, weil der Vorteil darüber hinaus für die medizinische oder pharmazeutische Praxis von Belang sein muss. Diese Voraussetzung ist erfüllt, wenn ein ausreichend direkter Bezug zur medizinischen oder

78 Eggenberger Stöckli, Arzt (Fn. 27), 486; Saxer, BSK HMG (Fn. 27), Art. 33 N 42; ders., Vorteilsverbot (Fn. 28), $133 \mathrm{f}$.

79 Bundesamt für Sozialversicherung (BSV), Empfehlung vom 21.12.2001, 2; dass., Empfehlung vom 15.3.2002, 1; dass., Empfehlung vom 11.7.2002, 1; Swissmedic, Swissmedic Journal 2006 (Fn. 27), 37.

80 BGE 140 II 520, 534 E. 5.2.4; Vasella (Fn. 26), 106.

81 Eichenberger/Marti/Straub, recht 2003 (Fn. 64), 227; Vasella (Fn. 26), 106.

82 Siehe zu diesen und weiteren Beispielen: Saxer, BSK HMG (Fn. 27), Art. 33 N 36 und 43; ders., AJP 2002 (Fn. 28), 1471; Vasella (Fn. 26), 106 f. pharmazeutischen Praxis des bevorteilten Leistungserbringers besteht. 83 Unzulässig sind daher Zuwendungen, die allein der Medizinalperson persönlich zugutekommen. ${ }^{84}$ Das Kriterium des Praxisbezugs wird von den Behörden allerdings eher grosszügig ausgelegt. 85

62 In Art. 33 Abs. 3 lit. b HMG findet sich die «zentrale Ausnahme» 86 für bestimmte Rabatte:

63 Unter Rabatten versteht man Preisnachlässe bzw. Vergünstigungen und andere finanzielle Vorteile, welche im Rahmen bestimmter Produktelieferungen auf den üblichen Verkaufspreis bzw. auf den jeweiligen Rechnungsbetrag eingeräumt werden. ${ }^{87}$ Rabatte können auf verschiedene Art und Weise vorkommen, etwa als fixer Betrag oder Prozentsatz des Bruttopreises. ${ }^{88}$ Man unterscheidet u.a. Mengen-, Barzahlungs- und Naturalrabatte sowie Skonti. 89 Das Gewähren und An-

83 Vgl. Urteil des Bundesverwaltungsgerichts C669/2008 vom 17.12.2010, E. 4.8.1; Urteil C4724/2010 vom 10.3.2014, E. 5.3.

84 BGE 140 II 520, 534 E. 5.2.4; Urteil des Bundesverwaltungsgerichts C-4724/2010 vom 10.3.2014, E. 5.3; Swissmedic, Swissmedic Journal 2006 (Fn. 27), 30.

85 Vgl. Swissmedic, Swissmedic Journal 2006 (Fn. 27), 38 Fn. 53 (m.w.H.), weshalb sozialadäquate Geschenke nicht geahndet werden. Siehe auch Eggenberger Stöckli, Arzt (Fn. 27), 486; dies., PharmR 2008, 459.

86 Vasella (Fn. 26), 112; siehe auch Saxer, AJP 2002 (Fn. 28), 1471; ders., BSK HMG (Fn. 27), Art. 33 $\mathrm{N} 44$.

87 BGE 140 II 520, 534 E. 5.2.4; Urteil des Bundesverwaltungsgerichts C-4724/2010 vom 10.3.2014, E. 3.6.2; Swissmedic, Swissmedic Journal 2003 (Fn. 27), 981.

88 Swissmedic, Swissmedic Journal 2003 (Fn. 27), 981 (m.w.H.); Vasella (Fn. 26), 113.

89 Vgl. Urteil des Bundesverwaltungsgerichts C4724/2010 vom 10.3.2014, E. 3.6.2; Eggenberger Stöckli, PharmR 2007 (Fn. 36), 394; Saxer, BSK HMG (Fn. 27), Art. 33 N 45; Swissmedic, Swissmedic Journal 2003 (Fn. 27), 981; Vasella (Fn. 26), 113 . 
nehmen von Rabatten ist dem Grunde nach verboten und nur unter den engen Voraussetzungen der Ausnahmevorschrift zulässig.

64 Als handelsüblich gelten Rabatte, wenn sie im Rahmen von Geschäftsbeziehungen zwischen Herstellern oder Lieferanten von Arzneimitteln und Leistungserbringern während eines gewissen Zeitraums erfolgen, sodass die Käufer auf den Preisnachlass vertrauen bzw. ihn auch für künftige Bestellungen als selbstverständlich ansehen.90 Die Abnehmer fassen darum den Nettopreis, d.h. den Bruttopreis abzüglich des handelsüblichen Rabatts, als Grundpreis auf, sodass der Nachlass aufgrund seiner Vorhersehbarkeit keinen Einfluss auf ihre Kaufentscheidung hat. ${ }^{1}$

65 Als betriebswirtschaftlich gerechtfertigt werden von Swissmedic solche Rabatte angesehen, die einem Wirtschaftsakteur ermöglichen, sich einen bestimmten Markt zu erschliessen bzw. sich den Bedingungen eines bestimmten Marktes anzupassen oder die Wettbewerbsfähigkeit seiner Produkte sicherzustellen..$^{2}$ Es kann sich dabei um einen ausserordentlich umfangreichen Rabatt handeln, den ein Hersteller im Rahmen der Markteinführung eines Produktes einräumt, oder um einen vorübergehenden Rabatt, der anlässlich des Auftretens ei-

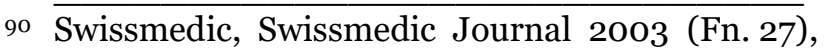
981 f.; vgl. auch Saxer, AJP 2002 (Fn. 28), 1472.

91 Swissmedic, Swissmedic Journal 2003 (Fn. 27), 981 f.; siehe auch Eggenberger Stöckli, Werbung (Fn. 49), 69.

92 Swissmedic, Swissmedic Journal 2003 (Fn. 27), 982; zustimmend Straub, Die Arzneimittelwerbung der Pharmaunternehmungen im regulatorischen Umfeld, in: Schaffhauser/Poledna (Hrsg.), Wettbewerb im Gesundheitsrecht, St. Gallen 2004, 55 ff., 66 f. nes neuen Konkurrenten etabliert wird. Demgegenüber erkennt die Lehre nur solche Rabatte als betriebswirtschaftlich gerechtfertigt an, denen im weiteren Sinne eine wirtschaftliche Gegenleistung des Empfängers gegenübersteht. 93

\section{b) Anwendbarkeit im TCM-Bereich}

66 Es ist zu prüfen, ob und wann die vorstehend dargestellten Ausnahmen vom heilmittelrechtlichen Vorteilsverbot in den Fällen der Erfassungsentschädigungen sowie der pauschalen Vergütungen und Zeilenhonorare greifen.

67 Eine Ausnahme für Vorteile von bescheidenem Wert nach Art. 33 Abs. 3 lit. a HMG liegt nicht vor:

68 Bei direkten Zahlungen wie den pauschalen Vergütungen und Zeilenhonoraren an Ärzte, mögen sie auch im Einzelfall geringwertig ausfallen, besteht kein (direkter) Bezug zur medizinischen oder therapeutischen Praxis der Fachperson.94 Da für Rabatte und andere Preisnachlässe mit Art. 33 Abs. 3 lit. b HMG ein eigener Erlaubnistatbestand geschaffen wurde, sind Zuwendungen wie die Erfassungsentschädigungen zu Gunsten der verschreibungs- bzw. abgabeberechtigten Leistungserbringer ausschliesslich nach jenen Ausnahmevoraussetzungen zu prüfen, und zwar unabhängig von der Höhe der eingeräumten Vergünstigung. 95

93 Vgl. Eggenberger Stöckli, Arzt (Fn. 27), 487; dies., Werbung, 69; Saxer, AJP 2002 (Fn. 28), 1472; ders., BSK HMG (Fn. 27), Art. 33 N 47.

94 Vgl. BGE 140 II 520, 534 E. 5.3.4; Saxer, BSK HMG (Fn. 27), Art. 33 N 43.

95 Vgl. Urteil des Bundesverwaltungsgerichts C669/2008 vom 17.12.2010, E. 4.8.1; Urteil C1663/2007 vom 28.6.2011, E. 4.1.2; Vasella (Fn. 26), 110. 
69 Die Ausnahme für handelsübliche oder betriebswirtschaftlich gerechtfertigte Rabatte gemäss Art. 33 Abs. 3 lit. b HMG ist in beiden Sachverhaltsvarianten ebenfalls nicht einschlägig:

$7 \mathbf{0}$ In der ersten Variante (Erfassungsentschädigungen) kommt die Ausnahmeregelung von Vornherein nur bei periodischen Abrechnungen (z.B. bei monatlicher oder halb-/jährlicher Abrechnung) gegenüber den Ärzten und Therapeuten in Betracht. Denn bei Sofortrechnungen direkt gegenüber den Patienten bzw. ihren Versicherungen liegt kein Rabattsystem vor, da ein eigentlicher Rabatt im Sinne eines Preisnachlasses zu Gunsten der Leistungserbringer in diesen Fällen gar nicht vorgesehen ist. 96

71 Bei periodischen Abrechnungen ist für die rechtliche Beurteilung im Hinblick auf die Handelsüblichkeit von Bedeutung, wie lange den Leistungserbringern die Rabatte für TCM-Präparate schon eingeräumt werden. Denn nur wenn diese Preisnachlässe bereits seit einigen Jahren die Geschäftsbeziehungen zu den Ärzten bzw. Therapeuten prägen, sodass jene mittlerweile wie selbstverständlich von einem Rabatt auf TCM-Arzneimittel ausgehen und ihre Bestellungen bei den Herstellern nicht aufgrund der Vergünstigungen tätigen, kann von Handelsüblichkeit ausgegangen werden.

72 Im Hinblick auf die Alternative der betriebswirtschaftlichen Rechtfertigung kann bei periodischen Abrechnungen wiederum auf das Argument verwiesen werden, dass sich der Aufwand der Ärzte bzw. Therapeuten bei der OnlineBestellung eher gering ausnimmt. Es

Vgl. BGE 140 II 520, 534 E. 5.3.4. fehlt damit an einer angemessenen wirtschaftlichen Gegenleistung.

73 Die Ausnahmeregelung gemäss Art. 33 Abs. 3 lit. b HMG ist in der zweiten Variante (pauschale Vergütungen und Zeilenhonorare) nicht einschlägig, da den Ärzten kein eigentliches Rabattsystem mit Preisnachlässen auf Arzneimittel offeriert wird. Hier werden keine Rabatte im Sinne einer Reduktion des Verkaufspreises bzw. des Rechnungsbetrages im Rahmen einer bestimmten Produktelieferung eingeräumt, sondern die Ärzte werden allgemein für das Verschreiben von TCM-Arzneimitteln und unabhängig von einer bestimmten Lieferung pauschal vergütet. Diese pauschalen Vergütungen sind allein davon abhängig, ob die Rezepte in einer bestimmten Apotheke eingelöst werden, stellen aber keine Preisnachlässe im Sinne des Ausnahmetatbestands dar.

\section{Heilmittelrechtliches Integritäts- und Transparenzgebot de lege ferenda}

74 Das heilmittelrechtliche Vorteilsverbot wird künftig zwei verschiedene Einzelregelungen umfassen: ein Integritäts- und ein Transparenzgebot (Art. 55 und 56 revHMG).

75 Verstösse können dereinst - abgesehen von den weiterhin geltenden Verwaltungsmassnahmen (Art. 66 revHMG) ausdrücklich nach einem verschärften Straftatbestand sanktioniert werden (Art. 86 Abs. 1 lit. h und Abs. 4 sowie 87 Abs. 1 lit. h und 3 revHMG). Die derzeitige Rechtslage, wonach es laut Bundesgericht keine ausreichend bestimmte Rechtsgrundlage für Strafmassnahmen 
gibt, 97 wird damit aufgehoben. Bei Zuwiderhandlungen kann eine Freiheitsstrafe bis zu drei Jahren oder eine Geldstrafe (bei einem Verstoss gegen das Integritätsgebot) bzw. eine Busse bis zu 50'0oo CHF (bei einem Verstoss gegen das Transparenzgebot) verhängt werden.

Die spezifischen Vorgaben im Heilmittelgesetz werden in Zukunft konkretisiert durch verschiedene Ausfiihrungsbestimmungen auf Verordnungsbasis. Von Bedeutung sind im vorliegenden $\mathrm{Zu}-$ sammenhang vor allem die neue Verordnung über die Integrität und Transparenz im Heilmittelbereich (E-VITH), die revidierte Arzneimittelverordnung (ErevVAM) und die revidierte Komplementär- und Phytoarzneimittelverordnung (E-revKPAV). 98

\section{Anwendungsbereich}

\section{a) Allgemeine Auslegung}

77 Der Anwendungsbereich des neu gefassten Integritätsgebots für den Arzneimittelvertrieb wird einerseits enger und andererseits weiter ausgestaltet sein als die heutige Verbotsnorm (Art. 55 Abs. 1 revHMG):

Die Vorschrift soll nur für verschreibungspflichtige Arzneimittel gelten, d.h. die Abgabekategorien $\mathrm{C}$ und $\mathrm{D}$ werden nicht mehr erfasst (die Abgabekategorie E bereits nicht nach geltendem Recht) 99 . Neben dem Verschreiben und Abgeben sollen allerdings auch das Anwenden und Einkaufen der rezeptpflichtigen Arzneimittel einen ausreichenden sachli-

97 Siehe Rz. 15.

98 Die Entwürfe dieser Verordnungen sind einsehbar auf der in Rz. 13 (Fn. 31) genannten Website.

$99 \mathrm{Zu}$ dieser Ausnahme siehe Rz. 18 und 45. chen Zusammenhang bilden. Zudem werden die Vorteile künftig als «nicht gebührend» beschrieben, so dass sie auch ideelle bzw. immaterielle Vorteile umfassen.

79 Dabei ist zu berücksichtigen, dass eine neue Einteilung der Abgabekategorien eingeführt werden soll (vgl. Art. 41-44 ErevVAM). ${ }^{100}$ Die Kategorie C wird ersatzlos gestrichen. Die gegenwärtig darin aufgelisteten Arzneimittel sollen zum Grossteil der Kategorie D und nur in Ausnahmen der Kategorie B zugeteilt werden. Demzufolge bleibt der überwiegende Teil der betreffenden Medikamente rezeptfrei und damit ausserhalb des Anwendungsbereichs von Art. 55 revHMG. ${ }^{101}$

8o Der Bundesrat kann die Gebotsregelung im neuen Verordnungsrecht auf weitere Heilmittel ausweiten (vgl. Art. 55 Abs. 3 revHMG), also wiederum auf nicht verschreibungspflichtige Arzneimittel. Die der Vernehmlassung zugrundeliegenden Entwürfe sehen keine entsprechenden Erweiterungen vor (vgl. insbesondere EVITH).

81 Nach derzeitigem Stand scheinen rezeptfreie Medikamente somit ausgenommen vom Integritätsgebot. Das legt jedenfalls der Wortlaut der neuen Bestimmung nahe, die sich in Abs. 1 explizit auf «ver-

$100 \overline{\mathrm{Zu} \text { den neuen Abgabekategorien siehe Bundes- }}$ amt für Gesundheit (BAG), Erläuterungen zur Arzneimittelverordnung (VAM), Bern 2017, 21 ff.; Mathys/Spörri, Projekt HMV IV, Informationsveranstaltung vom 6.4.2017, Bern 2017, passim; Vasella, Anpassungen bei der Arzneimittelabgabe, Swissblawg vom 23.4.2017, passim.

101 Vgl. Bundesamt für Gesundheit (BAG), Erläuterungen zur Verordnung über Integrität und Transparenz im Heilmittelbereich (VITH), Bern 2017, 11. 
schreibungspflichtige» Arzneimittel bezieht. Dabei handelt es sich im Ergebnis um eine abgeleitete bzw. sinngemässe Beschränkung des sachlichen Anwendungsbereichs durch die gesetzliche Umschreibung des persönlichen Geltungsbereichs, wonach die Vorteilsnehmer neu definiert werden als «Personen, die verschreibungspflichtige Arzneimittel verschreiben, abgeben, anwenden oder zu diesem Zweck einkaufen». Auch die Gesetzesmaterialien stellen diesbezüglich nur auf Medikamente der «Abgabekategorien A und B; vgl. Art. 23 Abs. 1 und 24 HMG» $a b,{ }^{102}$ was ebenfalls für einen Ausschluss von nicht verschreibungspflichtigen Präparaten spricht. In der parlamentarischen Debatte hiess es ausserdem, dass Vorteile beim Heilmitteleinkauf erlaubt sind, sofern sie im $\mathrm{Zu}-$ sammenhang mit nicht der Verschreibungspflicht unterliegenden Produkten gewährt bzw. angenommen werden.103 Der Bundesrat bestätigt diese Einschränkung in seinen Erläuterungen zum geplanten Ausführungsrecht. ${ }^{104}$

82 In Zukunft wird ferner, wie zur heutigen Rechtslage aufgezeigt, ${ }^{105}$ (weiterhin) nach der Verwendungsfertigkeit zu differenzieren sein. Letztlich dürften von der Gebotsnorm nur Arzneimittel erfasst werden, die sowohl verwendungsfertig als auch verschreibungspflichtig sind.

83 Zwar fänden sich wohl auch Argumente gegen eine Ausnahme vom Integritätsgebot. So liesse sich anführen, dass in den

102 Vgl. Botschaft Änderung HMG, BBl 2013, 78 (in Bezug auf Art. $57 a$ E-revHMG).

103 Vgl. Berset, Änderung Heilmittelgesetz, ABl 2016 SR, 33 (in Bezug auf Art. 57a Abs. 1 E-revHMG).

104 Vgl. BAG, Erläuterungen VITH, 17. Im Ergebnis ebenso Sprecher, ZSR 135 (2016) II (Fn. 19), 217. 105 Siehe Rz. 37 ff.
Erlaubnistatbeständen des Abs. 2 ganz generell von Heilmitteln die Rede ist (vgl. lit. c: «Abgeltungen [...] bei Bestellungen und Lieferungen von Heilmitteln»; lit. d: «beim Heilmitteleinkauf gewährte Preisrabatte oder Rückvergütungen»), weshalb auch von der Gebotsvorschrift des Abs. 1 e contrario global alle Arzneimittel - ungeachtet einer Verschreibungspflicht - erfasst sein müssten. Es ist allerdings zu vermuten, dass es sich bei dieser Formulierung der Erlaubnistatbestände entgegen dem klaren Wortlaut der sich allein auf rezeptpflichtige Medikamente beschränkenden Gebotsregelung - um ein gesetzgeberisches Versehen handelt, das dem intensiven und kontroversen Ringen um die konkrete Ausgestaltung der Norm während der langwierigen parlamentarischen Beratungen geschuldet ist. ${ }^{106}$

84 Das neu eingeführte Transparenzgebot enthält eine neue Verpflichtung, die eine Weitergabe von Vergünstigungen in Form jeglicher «Preisrabatte und Rückvergütungen» an die Patienten bzw. deren Versicherungen gewährleisten soll (Art. 56 Abs. 1 revHMG):

85 Bereits nach heutiger Rechtslage besteht eine Weitergabepflicht. Die bevorteilten Leistungserbringer müssen die direkten oder indirekten Vergünstigungen, die sie im Rahmen einer durch die obligatorische Krankenpflegeversicherung gedeckten Behandlung erhalten, an ihre Patienten bzw. deren Versicherer weitergeben (vgl. Art. 56 Abs. 3 und 4 KVG). ${ }^{107}$

$106 \overline{\mathrm{Zu} \text { diesem Gesetzgebungsverfahren siehe Vasella }}$ (Fn. 26), $583 \mathrm{ff}$.

107 Mit Inkrafttreten der Reform des Heilmittelrechts können Versicherer und Leistungserbringer allerdings vereinbaren, dass die Vergünstigungen nicht vollumfänglich weitergegeben wer- 
Nach Art. 56 revHMG bestehen weitreichende Dokumentations- und Offenlegungspflichten. Es sind sämtliche beim Heilmitteleinkauf gewährten Preisrabatte und Rückvergütungen (i) in den Belegen, Rechnungen und Geschäftsbüchern der verkaufenden und der einkaufenden Vertragspartei auszuweisen sowie (ii) den zuständigen Behörden auf Verlangen offenzulegen.

87 Die Bestimmung soll nach ihrem Wortlaut für alle Heilmittel gelten. Für Arzneimittel (und Medizinprodukte) mit geringem Risikopotenzial kann der Bundesrat verordnungsrechtliche Ausnahmen vorsehen (vgl. Art. 56 Abs. 3 revHMG). ${ }^{108}$

\section{b) Anwendbarkeit im TCM-Bereich}

88 Arzneimittel aus der Produktpalette der Komplementär- und Phytomedizin, wozu die TCM-Präparate auch weiterhin zählen (vgl. Art. 4 Abs. 4 lit. a revKPV), werden als OTC ${ }^{109-M e d i k a m e n t e ~ n a c h ~ d e r ~}$ hier vertretenen Auffassung im Regelfall nicht vom Integritätsgebot erfasst sein. Denn die verwendungsfertigen TCMSpezialitäten gehören - sowohl nach aktueller Einteilung (Abgabekategorie C) als auch nach geplanter Umteilung ( $A b$ gabekategorie D) - fast ausschliesslich zu den nicht verschreibungspflichtigen Arzneimitteln (vgl. TAS-Liste). ${ }^{110}$ Die geplante Reform dürfte für den Grossteil des TCM-Bereichs insoweit eine klare

den müssen, sofern davon weniger als die Hälfte einbehalten wird und dieser zurückbehaltene Betrag nachweislich zur Verbesserung der Behandlungsqualität eingesetzt wird (vgl. Art. 56 Abs. $3^{\text {bis }}$ revKVG).

108 Siehe dazu Rz. 96.

109 Abkürzung für «over the counter» (dt.: «über den Ladentisch»).

110 Siehe dazu Rz. 18, 30 und 79.
Regelung und legislatorische Lockerung bringen, als dass Art. 55 Abs. 1 revHMG nach der dargelegten Einschätzung keine Anwendung auf rezeptfreie Arzneimittel finden soll. Diese tatbestandliche Einschränkung setzt allerdings voraus, dass die endgültige Fassung des ausführenden Verordnungsrechts den sachlichen Geltungsbereich der Gesetzesbestimmung nicht auf alle Arzneimittel (oder gar alle Heilmittel) und damit auch auf rezeptfreie Medikamente erweitert.

89 Für die wenigen TCM-Präparate, die zur Abgabekategorie B zählen, kann für die Anwendbarkeit des Integritätsgebots auch nach künftiger Rechtslage auf die oben geführte Argumentation zu Art. 33 HMG abgestellt werden. ${ }^{111}$ Das gilt insbesondere für die verwendungsfertigen TCM-Magistralrezepturen, die einer «doppelten» ärztlichen Verschreibung bedürfen, und zwar sowohl für die Herstellung als auch für die Abgabe (vgl. Art. 9 Abs. 2 lit. a HMG).

90 TCM-Präparate sind Heilmittel mit vorwiegend geringem Risikopotential, weshalb sie von Swissmedic mehrheitlich in die (neuen) Abgabekategorien $\mathrm{D}$ und $\mathrm{E}$ eingeteilt werden dürften. Nachdem die Dokumentations- und Offenlegungspflichten jedoch explizit für sämtliche beim Heilmitteleinkauf gewährten Preisrabatte und Rückvergütungen gelten sollen, kommen auch für alle TCMArzneimittel grundsätzlich keine Sonderregeln vom Transparenzgebot in Betracht. ${ }^{112}$

$111 \overline{\text { Siehe dazu Rz. 26, } 28 \text { ff. und } 31 \text { ff., } 37 \text { ff. und }}$ $42 \mathrm{ff}$.

112 Es bestehen allerdings Ausnahmen von diesem Gebot; siehe dazu Rz. 91 ff. und 97 ff. 


\section{Ausnahmetatbestände}

\section{a) Allgemeine Auslegung}

91 Es sind bestimmte Ausnahmen vom Integritätsgebot vorgesehen, die weitere bzw. weiter gefasste Konstellationen beinhalten als der heutige Ausnahmekata$\log$ und im Detail durch Verordnungen des Bundesrates geregelt werden können. Von Interesse sind im vorliegenden Kontext die folgenden Erlaubnistatbestände (Art. 55 Abs. 2 revHMG):

Zulässig bleiben Vorteile von bescheidenem Wert, die für die medizinische oder pharmazeutische Praxis von Belang sind (lit. a). ${ }^{113}$ Darunter fallen gemäss der ausdrücklichen Neuregelung nur Vorteile im Gesamtwert von höchstens $300 \mathrm{CHF}$ pro Fachperson und Jahr (vgl. Art. 3 Abs. $1 \mathrm{E}$-VITH). Sie sind für die medizinische oder pharmazeutische Praxis dann von Belang, wenn sie entweder in direktem Zusammenhang mit der Berufsausübung der Fachperson stehen oder direkt der Kundschaft der Fachperson zugutekommen (vgl. Art. 3 Abs. 2 E-VITH).

Zudem sollen Abgeltungen für Gegenleistungen, etwa bei der Bestellung und Lieferung von Heilmitteln, dann nicht vom Integritätsgebot erfasst sein, wenn sie gleichwertig ausgestaltet sind (lit. c). ${ }^{114}$ Das soll unter bestimmten Voraussetzungen der Fall sein (vgl. Art. 7 Abs. 1 E-VITH). Die Abgeltung muss auf einer schriftlichen Vereinbarung basieren, die Art und Umfang von Gegenleistung und Abgeltung festhält. Zulässig ist die $\mathrm{Zu}-$ wendung nur für eine Gegenleistung, die der Fachperson oder Organisation einen

113 Siehe dazu BAG, Erläuterungen VITH, 12 f.

114 Siehe dazu BAG, Erläuterungen VITH, $16 \mathrm{f}$. zusätzlichen, über gesetzliche Verpflichtungen hinausgehenden Aufwand verursacht, nicht anderweitig vergütet wird und der Fachperson oder Organisation keinen direkten Nutzen bringt. Schliesslich haben Umfang und Aufwand der Gegenleistung ungefähr dem Wert der Abgeltung zu entsprechen, d.h. die Leistungen beider Parteien müssen nach marktwirtschaftlichen Kriterien in einem angemessenen Verhältnis zueinanderstehen.

94 Ebenfalls zulässig sind künftig die beim Heilmitteleinkauf gewährten Preisrabatte und Rückvergütungen, sofern sie keinen Einfluss auf die Wahl der Behandlung haben (lit. d). ${ }^{115}$ Davon soll dann auszugehen sein, wenn sie den Patienten bzw. Konsumenten direkt oder indirekt zugutekommen (vgl. Art. 8 Abs. 1 EVITH).

95 Ferner sind Ausnahmen vom Transparenzgebot für Heilmittel mit geringem Risikopotenzial geplant, für die eine $\mathrm{Ne}-$ gativliste erstellt wurde (Art. 56 Abs. 3 revHMG):

96 Einen Dispens von den Dokumentationsund Offenlegungspflichten hat der Bundesrat verordnungsrechtlich u.a. für den Einkauf von frei verkäuflichen Arzneimitteln vorgesehen (vgl. Art. 10 Abs. 2 EVITH). Damit wird das neue Transparenzgebot zwar grundsätzlich auch rezeptfreie Medikamente erfassen, aber nach dem revidierten Heilmittelrecht ausdrücklich - nicht diejenigen der Abgabekategorie E. ${ }^{116}$

\footnotetext{
115 Siehe dazu BAG, Erläuterungen VITH, $17 \mathrm{f}$.

${ }_{116} \mathrm{Zu}$ den Abgabekategorien siehe Rz. 18 (mit Fn. 35).
} 


\section{b) Anwendbarkeit im TCM-Bereich}

97 Nachdem keine besonderen Ausnahmeregelungen für TCM-Arzneimittel zu erwarten sind, gelten im Hinblick auf das Integritätsgebot die allgemeinen Erlaubnistatbestände:

98 Für die Vorteile von bescheidenem Wert kann auf die Erläuterungen zur geltenden Rechtslage verwiesen werden, ${ }^{117}$ da im Wesentlichen einfach die in der bisherigen Praxis herausgearbeiteten Kriterien kodifiziert werden. ${ }^{118}$

99 Was die Abgeltungen für Gegenleistungen betrifft, wird im konkreten Einzelfall $\mathrm{zu}$ entscheiden sein, ob die jeweiligen Vorteile gleichwertig sind. In Betracht kommt die Ausnahme nur bei Variante 1 (Erfassungsentschädigungen), da es bei Variante 2 (pauschale Vergütungen und Zeilenhonorare) bereits an einer Gegenleistung fehlt. Im Lichte der Ausführungen zum heutigen Recht ist insbesondere kritisch zu prüfen, ob durch die OnlineBestellungen von TCM-Präparaten mittels spezieller Software tatsächlich ein über die gesetzlichen Pflichten hinausgehender Aufwand entsteht, der nicht anderweitig (z.B. über den TARMED) vergütet wird und in einem angemessenen Verhältnis zur Gegenleistung steht. In den Gesetzesmaterialien findet sich der Hinweis, dass es sich bei der Übermittlung von ärztlichen Rezepten auf elektronischem Weg um einen Aufwand handle, der den Fachpersonen oder Organisationen aufgrund der Vereinfachung von Arbeitsabläufen selbst einen direkten Nutzen bringe und deshalb nicht entschädigt werden dürfe. ${ }^{119}$

117 Siehe Rz. 59 ff.

118 Vgl. BAG, Erläuterungen VITH, 12 (m.w.H.).

119 Vgl. BAG, Erläuterungen VITH, 16.
100 Ebenfalls mit Bedacht wird man Fälle von Preisrabatten oder Rückvergütungen bewerten müssen, denn es sind bei den oben vorgestellten Varianten kaum Konstellationen denkbar, in denen nicht mindestens die Gefahr einer Einflussnahme auf die Wahl der jeweiligen Behandlung besteht. Denn diese Zahlungen können (jedenfalls unbewusst) auf die medizinisch-pharmazeutische Fachperson einwirken und ihre Entscheidung für bestimmte TCM-Präparate beeinflussen, ohne dass die Kick-backs der Hersteller den Patienten und Kunden der bevorteilten Leistungserbringer direkt oder indirekt zugutekommen. Vielmehr erlangen die Leistungserbringer selbst einen unmittelbaren Vermögensvorteil, der auf ihren Therapieentscheid einwirken könnte. ${ }^{120}$

101 Hinsichtlich des Transparenzgebots ist beim Vertrieb von TCM-Arzneimitteln, die neu ganz überwiegend in die Abgabekategorien D und $\mathrm{E}$ eingestuft werden, zu differenzieren:

102 Auch bei den im TCM-Bereich gezahlten Erfassungsentschädigungen bzw. pauschalen Vergütungen und Zeilenhonoraren werden künftig in aller Regel Dokumentations- und Transparenzpflichten einzuhalten sein; nur die frei verkäuflichen TCM-Präparate werden nicht vom Transparenzgebot erfasst sein. 


\section{Schlussfolgerungen}

\section{Zusammenfassung der Ergebnisse}

\section{a) Geltendes Recht}

103 Das heilmittelrechtliche Vorteilsverbot (Art. $33 \mathrm{HMG}$ ) gilt für alle verwendungsfertigen Arzneimittel, unabhängig davon, ob sie zugelassen bzw. zulassungspflichtig, verschreibungspflichtig oder kassenpflichtig sind. Die Verbotsnorm erfasst daher auch TCM-Präparate in verwendungsfertiger Form, und zwar sowohl TCM-Spezialitäten als auch TCMMagistralrezepturen. Ausnahmen bestehen lediglich für rezeptfreie Medikamente der Abgabekategorie E.

\section{b) Künftiges Recht}

104 Medikamente der TCM sind im Regelfall nicht rezeptpflichtig und werden nach neuem Recht ganz überwiegend zur Abgabekategorie D zählen. Deshalb ist das nur für verschreibungspflichtige Arzneimittel geltende Integritätsgebot (Art. 55 revHMG) auf TCM-Präparate nicht anwendbar.

105 Das Transparenzgebot (Art. 56 revHMG) wird hingegen den Vertrieb sämtlicher Heilmittel erfassen, weshalb die neue Regelung auch für den TCM-Bereich greift. Eine Ausnahme soll für die frei verkäuflichen Arzneimittel der Abgabekategorie E gelten.

\section{Handlungsempfehlungen für die Praxis}

106 Die aufgezeigten (aktuellen und künftigen) rechtlichen Risiken, die beim Vertrieb von TCM-Arzneimitteln im Hinblick auf das heilmittelrechtliche Vorteilsverbot bzw. das heilmittelrechtliche
Integritäts- und Transparenzgebot bestehen, lassen sich mit zielgerichteten Massnahmen minimieren:

107 1. Beschränken von Erfassungsentschädigungen: Abgeltungen für OnlineBestellungen sollten bis zum Inkrafttreten des revidierten Heilmittelgesetzes nur sehr gering ausfallen und dürfen danach nur für rezeptfreie Medikamente vereinbart und ausgerichtet werden. Eine allgemeine Empfehlung zur maximalen Höhe ist mangels gefestigter Behördenund Gerichtspraxis nicht möglich. Die Limite kann nur im konkreten Einzelfall und gestützt auf eine interne Kostenanalyse erfolgen. Es muss nachweisbar sein, dass die elektronische Ausstellung eines Rezepts gegenüber der schriftlichen Verschreibung einen Mehraufwand bedeutet und dass dieser Mehraufwand der Leistungserbringer nicht bereits durch den TARMED abgedeckt ist.

2. Keine pauschalen Vergütungen oder Zeilenhonorare: Solche Abgeltungen in pauschalierter Form dürfen weder nach geltender noch nach künftiger Rechtslage vorgenommen werden.

3. Beachten der Weitergabepflicht: Die Leistungserbringer auf der Nehmerseite müssen ihrer Verpflichtung nachkommen, Vergünstigungen auf TCMArzneimittel von der SL-Arzneimittelliste an ihre Patienten bzw. deren Versicherer weiterzugeben. Sie können mit den Krankenversicherungen jeweils Vereinbarungen über den Einbehalt eines Teils der Vergünstigungen treffen. Die Arzneimittelhersteller auf der Geberseite sollten ihre Vertragspartner in geeigneter Weise auf diese Weitergabepflicht hinweisen. Solch ein (schriftlicher) Hinweis 
kann etwa in Produkt- und Dienstleistungskatalogen, in Newslettern, auf Werbeflyern oder auf der Website erfolgen.

110 4. Dokumentation und Transparenz: Mit Blick auf die bevorstehende Gesetzesrevision sollten alle Preisrabatte und Rückvergütungen in Belegen, Rechnungen und Geschäftsbüchern nachvollziehbar (d.h. korrekt, klar und vollständig) ausgewiesen werden. Diese Unterlagen sind zehn Jahre ${ }^{121}$ aufzubewahren, damit sie den zuständigen Behörden auf Verlangen für den massgeblichen Zeitraum ausgehändigt werden können.

111 5. Anpassen der Compliance: Die eigenen Verträge und Vertriebsstrategien sowie die internen Richtlinien im $\mathrm{Zu}$ sammenhang mit TCM-Arzneimitteln sollten überprüft und allenfalls im Hinblick auf das geplante Inkrafttreten des revidierten Heilmittelrechts bis spätestens Ende 2018 angepasst werden.

121 Dieser Zeitraum orientiert sich an der obligationenrechtlichen Pflicht zur Aufbewahrung bilanzwirksamer Dokumente nach Art. 958f Abs. 1 OR; vgl. Swissmedic, Swissmedic Journal 2010 (Fn. 65), 31. Siehe auch Vasella (Fn. 26), 475. 